\title{
LYUSTERNIK-GRAVES THEOREMS FOR THE SUM OF A LIPSCHITZ FUNCTION AND A SET-VALUED MAPPING*
}

\author{
R. CIBULKA $^{\dagger}$, A. L. DONTCHEV $\ddagger$, AND V. M. VELIOV
}

\begin{abstract}
In a paper of 1950 Graves proved that for a function $f$ acting between Banach spaces and an interior point $\bar{x}$ in its domain, if there exists a continuous linear mapping $A$ which is surjective and the Lipschitz modulus of the difference $f-A$ at $\bar{x}$ is sufficiently small, then $f$ is (linearly) open at $\bar{x}$. This is an extension of the Banach open mapping principle from continuous linear mappings to Lipschitz functions. A closely related result was obtained earlier by Lyusternik for smooth functions. In this paper, we obtain Lyusternik-Graves theorems for mappings of the form $f+F$, where $f$ is a Lipschitz continuous function around $\bar{x}$ and $F$ is a set-valued mapping. Roughly, we give conditions under which the mapping $f+F$ is linearly open at $\bar{x}$ for $\bar{y}$ provided that for each element $A$ of a certain set of continuous linear operators the mapping $f(\bar{x})+A(\cdot-\bar{x})+F$ is linearly open at $\bar{x}$ for $\bar{y}$. In the case when $F$ is the zero mapping, as corollaries we obtain the theorem of Graves as well as open mapping theorems by Pourciau and Páles, and a constrained open mapping theorem by Cibulka and Fabian. From the general result we also obtain a nonsmooth inverse function theorem proved recently by Cibulka and Dontchev. Application to Nemytskii operators and a feasibility mapping in control are presented.
\end{abstract}

Key words. open mapping theorem, inverse function theorem, linear openness, metric regularity, strict prederivative, feasibility in control

AMS subject classifications. 46A30, 47J07, 47H04, 49J52, 49J53

DOI. $10.1137 / 16 \mathrm{M} 1063150$

1. Introduction. Given a bounded linear mapping $A$ acting between Banach spaces $X$ and $Y$, the Banach open mapping principle says that the following three conditions are equivalent:

(i) $A$ is surjective;

(ii) $A$ is open at any $x \in X$, meaning that for every neighborhood $U$ of $x, A U$ is a neighborhood of $A x$;

(iii) there exists a constant $\tau>0$ such that $d\left(x, A^{-1}(y)\right) \leq \tau\|y-A x\|$ for all $x \in X, y \in Y$.

The conditions (ii) and (iii) remain the same if one sets $x=0$ in them. The condition (iii) can also be written as

$$
\left\|A^{-1}\right\|^{-}<\infty
$$

where $\|\cdot\|^{-}$denotes the inner norm. Recall that for a positively homogeneous setvalued mapping $H: Y \rightarrow X$ the inner norm is defined as

\footnotetext{
* Received by the editors February 25, 2016; accepted for publication (in revised form) August 25, 2016; published electronically December 14, 2016.

http://www.siam.org/journals/sicon/54-6/M106315.html

Funding: The first author was supported by project GA15-00735S. The second author was supported by Austrian Science Foundation (FWF) grant P26640-N25 and Australian Research Council Project DP160100854. The third author was supported by FWF grant P26640-N25.

${ }^{\dagger}$ NTIS-New Technologies for Information Society and Department of Mathematics, Faculty of Applied Sciences, University of West Bohemia, Technická 8, Pilsen, Czech Republic (cibi@kma.zcu.cz).

${ }^{\ddagger}$ Mathematical Reviews, Ann Arbor, MI 48107-8604 (ald@ams.org).

$\S$ Institute of Statistics and Mathematical Methods in Economics, Vienna University of Technology, Wiedner Hauptstrasses 8, A-1040 Vienna (veliov@tuwien.ac.at).
} 


$$
\|H\|^{-}:=\sup _{\|y\| \leq 1} \inf _{x \in H(y)}\|x\|
$$

(with the usual convention that $\inf \emptyset=\infty$ and, as we work with nonnegative quantities, that $\sup \emptyset=0$ ).

In the statements above and further in the paper we use following notations. When we write $f: X \rightarrow Y$ we mean that $f$ is a (single-valued) function acting from $X$ to $Y$, while $F: X \rightrightarrows Y$ is a mapping from $X$ to $Y$ which may be set-valued. We restrict our attention to Banach spaces $X$ and $Y$ with norms $\|\cdot\|$ although some of the results are valid for more general (metric) spaces. In any space the closed ball with center $a$ and radius $r$ is denoted by $\mathbb{B}_{r}(a)$, the corresponding open ball is $\underset{\mathbb{B}_{r}}{ }(a)$, the closed unit ball is $\mathbb{B}$, and the open one is $\mathbb{B}$. The graph of $F: X \rightarrow Y$ is the set gph $F=\{(x, y) \in$ $X \times Y \mid y \in F(x)\}$, the domain of $F$ is $\operatorname{dom} F=\{x \in X \mid F(x) \neq \emptyset\}$, and the inverse of $F$ is the mapping $y \mapsto F^{-1}(y)=\{x \in X \mid y \in F(x)\}$. We denote by $d(x, C)$ the distance from a point $x \in X$ to a set $C \subset X$, that is, $d(x, C):=\inf \{\|x-v\| \mid v \in C\}$. The radius of a set $C$ is defined as $\operatorname{rad}(C)=\inf _{x \in C} \sup _{y \in C}\|x-y\|$. The excess from a set $C$ to a set $D$ is $e(C, D)=\sup _{x \in C} d(x, D)$. The space of all linear bounded mappings acting from $X$ to $Y$ equipped with the standard operator norm is denoted by $\mathcal{L}(X, Y)$. The Lipschitz modulus of a function $f: X \rightarrow Y$ at $\bar{x} \in \operatorname{int} \operatorname{dom} f$ is defined as

$$
\operatorname{lip}(f ; \bar{x}):=\limsup _{\substack{x, x^{\prime} \rightarrow \bar{x} \\ x \neq x^{\prime}}} \frac{\left\|f(x)-f\left(x^{\prime}\right)\right\|}{\left\|x-x^{\prime}\right\|} .
$$

The condition $\operatorname{lip}(f ; \bar{x})<\infty$ means that $f$ is Lipschitz continuous in a neighborhood of $\bar{x}$; more precisely, for any $\ell>\operatorname{lip}(f ; \bar{x})$ there exists a neighborhood $U$ of $\bar{x}$ such that $f$ is Lipschitz continuous on $U$ with the constant $\ell$.

Graves published in [17] a theorem whose (slightly updated) statement is as follows.

TheOrem 1 (Graves (1950)). Consider a function $f: X \rightarrow Y$ along with a point $\bar{x} \in \operatorname{int} \operatorname{dom} f$. Suppose that there exist positive constants $\kappa$ and $\mu$ with $\kappa \mu<1$ and a bounded linear mapping $A: X \rightarrow Y$ such that

$$
\operatorname{lip}(f-A ; \bar{x}) \leq \mu \quad \text { and } \quad\left\|A^{-1}\right\|^{-} \leq \kappa .
$$

Then for any sufficiently small $\varepsilon>0$ one has

$$
f(\bar{x}+\varepsilon \mathbb{B}) \supset f(\bar{x})+\left(\kappa^{-1}-\mu\right) \varepsilon \mathbb{B} .
$$

Note that the linear and bounded mapping $A$ in Theorem 1 may be not unique but if there are two such mappings they should be "not too far" from each other; we will go further with this observation in Theorem 6 given later in this section. For $f=A$ Theorem 1 yields the Banach open mapping principle; indeed, in that case $\bar{x}$ could be any point in $X$ and $\mu$ could be any positive real less than $1 / \kappa$. Furthermore, if $\mu$ could be arbitrarily small, then $A$ is the (unique) strict derivative of $f$ at $\bar{x}$. Dontchev observed in [11] (see also [12, section 5.4]) that the proof of Graves in [17] can be easily adjusted to imply a property of the function $f$ stronger than the one in (3); here we employ this property in the following form: for $f: X \rightarrow Y$ and $\bar{x} \in \operatorname{dom} f$ there are positive $\lambda$ and $\delta$ such that for each $x \in \mathbb{B}_{\delta}(\bar{x}) \cap \operatorname{dom} f$ and each $\varepsilon \in(0, \delta)$ we have

$$
f((x+\varepsilon \mathbb{B}) \cap \operatorname{dom} f) \supset[f(x)+\lambda \varepsilon \mathbb{B}] \cap \mathbb{B}_{\delta}(f(\bar{x})) .
$$


Property (4) is known as linear openness of $f$ around the point $\bar{x}$. The linear openness of $f$ around $\bar{x}$ is stronger than the (usual) openness of $f$ at $\bar{x}$ (for any neighborhood of $U$ of $\bar{x}, f(U)$ is a neighborhood of $f(\bar{x})$ ); these properties become equivalent for bounded linear mappings.

Condition (iii) in the Banach open mapping principle means that the mapping $A$ is metrically regular. In general, a mapping $F: X \rightrightarrows Y$ is said to be metrically regular at $\bar{x}$ for $\bar{y}$ when $\bar{y} \in F(\bar{x})$, gph $F$ is locally closed at $(\bar{x}, \bar{y})$, meaning that there exists a neighborhood $W$ of $(\bar{x}, \bar{y})$ such that the set gph $F \cap W$ is closed in $W$, and there is a constant $\tau \geq 0$ together with neighborhoods $U$ of $\bar{x}$ and $V$ of $\bar{y}$ such that

$$
d\left(x, F^{-1}(y)\right) \leq \tau d(y, F(x)) \quad \text { for every } \quad(x, y) \in U \times V .
$$

The infimum of all constants $\tau \geq 0$ such that (5) holds for some neighborhoods $U$ and $V$ is said to be the regularity modulus of $F$ at $\bar{x}$ for $\bar{y}$ and is denoted by $\operatorname{reg}(F ; \bar{x} \mid \bar{y})$. In short, metric regularity of $F$ at $\bar{x}$ for $\bar{y}$ is signaled by $\operatorname{reg}(F ; \bar{x} \mid \bar{y})<\infty$. In case of a single-valued function $f: X \rightarrow Y$ we use the shorter notation $\operatorname{reg}(f ; \bar{x})$ instead of $\operatorname{reg}(f ; \bar{x} \mid f(\bar{x}))$. In terms of metric regularity, the Banach open mapping principle says that a mapping $A \in \mathcal{L}(X, Y)$ is metrically regular at any point if and only if it is surjective, or open at any point, in which case $\operatorname{reg}(A ; 0)=\left\|A^{-1}\right\|^{-}$.

The property of linear openness of a function $f$ defined in (4) can be extended to a general set-valued mapping $F: X \rightrightarrows Y$ in the following way, with a slight abuse of notation. A mapping $F: X \rightrightarrows Y$ is said to be linearly open at $\bar{x}$ for $\bar{y}$ when $\bar{y} \in F(\bar{x})$, gph $F$ is locally closed at $(\bar{x}, \bar{y})$, and there exist neighborhoods $U$ of $\bar{x}$ and $V$ of $\bar{y}$ and a constant $\tau \geq 0$ such that

$$
F(x+\tau \varepsilon \mathbb{B}) \supset[F(x)+\varepsilon \mathbb{B}] \cap V \text { for all } x \in U \text { and all } \varepsilon>0 .
$$

There is a third property, introduced in 1981 by Aubin and named after him, which is equivalent to linear openness of the inverse. A mapping $S: Y \rightarrow X$ is said to have the Aubin property at $\bar{y}$ for $\bar{x}$ whenever $\bar{x} \in S(\bar{y})$, gph $S$ is locally closed at $(\bar{y}, \bar{x})$, and there exist a constant $\tau \geq 0$ and neighborhoods $U$ of $\bar{x}$ and $V$ of $\bar{y}$ such that for every $y, y^{\prime} \in V$ and every $x^{\prime} \in S\left(y^{\prime}\right) \cap U$ there exists $x \in S(y)$ with the property $\left\|x-x^{\prime}\right\| \leq \tau\left\|y-y^{\prime}\right\|$. In terms of the excess, this property becomes

$$
e\left(S\left(y^{\prime}\right) \cap U, S(y)\right) \leq \tau\left\|y-y^{\prime}\right\| \quad \text { for all } \quad y^{\prime}, y \in V .
$$

Starting with the groundbreaking works by Borwein and Zhuang [2] and Penot [24], it is well documented in the literature that metric regularity of a mapping $F$ at $\bar{x}$ for $\bar{y}$ is equivalent to the Aubin property of $F^{-1}$ at $\bar{y}$ for $\bar{x}$ as well as to the linear openness of $F$ at $\bar{x}$ for $\bar{y}$; moreover, the infimum of all constants $\tau \geq 0$ such that either (6) or (7) holds for some neighborhoods $U$ and $V$ equals $\operatorname{reg}(F ; \bar{x} \mid \bar{y})$. Later in the paper we use the known fact that if $f: X \rightarrow Y$ and $\bar{x} \in \operatorname{dom} f$, then $1 / \operatorname{reg}(f ; \bar{x})$ is equal to the supremum of all constants $\lambda \geq 0$ for which there is $\delta>0$ such that for each $x \in \mathbb{B}_{\delta}(\bar{x}) \cap \operatorname{dom} f$ and each $\varepsilon \in(0, \delta)$ the inclusion (4) is satisfied. In this paper we state the results in terms of metric regularity; clearly, they could be reformulated in terms of the linear openness or the Aubin property.

We present next the following generalization of Theorem 1 for set-valued mappings in Banach spaces, which is a particular case of [12, Theorem 5E.1].

Theorem 2 (extended Graves theorem). Consider a function $f: X \rightarrow Y, a$ set-valued mapping $F: X \rightrightarrows Y$, and a point $(\bar{x}, \bar{y}) \in \operatorname{gph}(f+F)$, along with positive 
constants $\kappa$ and $\mu$ such that $\kappa \mu<1$. Suppose that there exists a bounded linear mapping $A: X \rightarrow Y$ such that

$$
\operatorname{lip}(f-A ; \bar{x}) \leq \mu \quad \text { and } \quad \operatorname{reg}(f(\bar{x})+A(\cdot-\bar{x})+F(\cdot) ; \bar{x} \mid \bar{y}) \leq \kappa .
$$

Then

$$
\operatorname{reg}(f+F ; \bar{x} \mid \bar{y}) \leq\left(\kappa^{-1}-\mu\right)^{-1} .
$$

Note that by (8) we have $\bar{x} \in \operatorname{int} \operatorname{dom} f$. If $f$ is strictly Fréchet differentiable at $\bar{x}$ with derivative $D f(\bar{x})$, then we can choose $A=D f(\bar{x})$ in both Theorems 1 and 2 and then $\mu$ in (2) or (8) is just zero while $\kappa$ could be any real number greater than or equal to $\left\|[D f(\bar{x})]^{-1}\right\|^{-}$. In the case of a function, that is, for $F$ the zero mapping, we obtain that $f$ is metrically regular at $\bar{x}$ if and only if the derivative $D f(\bar{x})$ is surjective. This corollary of Theorem 1 was linked in Dmitruk, Milyutin, and Osmolovskii [13] to a theorem proved earlier by Lyusternik in [22], which involves differentiability in an essential way. Metric regularity, linear openness, and the Aubin property, as well as the theorems of Lyusternik and Graves and their role in modern analysis, have been broadly covered in the monographs [3], [9], [12], and [25]. A recent survey on this topic together with a rich bibliography can be found in [20].

More than two decades before his paper [17], Graves, together with Hildebrand, published in [18, Theorem 3] a nonsmooth inverse function theorem, the following slightly updated version of which is strikingly similar to Theorem 1.

Theorem 3 (Hildebrand and Graves (1927)). Consider a function $f: X \rightarrow X$ along with a point $\bar{x} \in \operatorname{int} \operatorname{dom} f$. Suppose that there exist positive constants $\kappa$ and $\mu$ with $\kappa \mu<1$ and a bounded linear mapping $A: X \rightarrow Y$ such that

$$
\operatorname{lip}(f-A ; \bar{x}) \leq \mu \quad \text { and } \quad\left\|A^{-1}\right\| \leq \kappa .
$$

Then for every $\ell>\left(\kappa^{-1}-\mu\right)^{-1}$ there exist neighborhoods $U$ of $\bar{x}$ and $V$ of $f(\bar{x})$ such that the mapping $V \ni y \mapsto f^{-1}(y) \cap U$ is a Lipschitz continuous function on $V$ with a Lipschitz constant $\ell$.

The property of the inverse $f^{-1}$ displayed in Theorem 3 means that $f^{-1}$ has a Lipschitz continuous single-valued graphical localization. In general, a mapping $T: Y \rightrightarrows X$ with $(\bar{y}, \bar{x}) \in \operatorname{gph} T$ is said to have a single-valued graphical localization around $\bar{y}$ for $\bar{x}$ when there are neighborhoods $U$ of $\bar{y}$ and $V$ of $\bar{x}$ such that the mapping $U \ni y \mapsto T(x) \cap V$ is single-valued on $U$. The property of existence of a Lipschitz single-valued graphical localization of the inverse implies metric regularity but is stronger than that and is called strong metric regularity. Generally, a mapping $F: X \rightrightarrows Y$ is said to be strongly metrically regular at $\bar{x}$ for $\bar{y}$ if $(\bar{x}, \bar{y}) \in \operatorname{gph} F$ and the inverse $F^{-1}$ has a Lipschitz continuous single-valued graphical localization around $\bar{y}$ for $\bar{x}$. It turns out that a mapping $F$ is strongly metrically regular at $\bar{x}$ for $\bar{y}$ if and only if it is metrically regular at $\bar{x}$ for $\bar{y}$ and the inverse $F^{-1}$ has a graphical localization around $\bar{y}$ for $\bar{x}$ which is nowhere multivalued (see [12, Proposition 3G.1]); moreover, for every single-valued localization $s$ of $F^{-1}$ around $\bar{y}$ for $\bar{x}$ one has $\operatorname{lip}(s ; \bar{y})=\operatorname{reg}(F ; \bar{x} \mid \bar{y})$. We will utilize the latter result later in the paper.

The property of strong metric regularity was coined by Robinson in his seminal paper [28], where he extended the paradigm of the inverse/implicit function theorem to "generalized equations" defined as inclusions of the form

$$
f(x)+F(x) \ni 0,
$$


where $f$ is a function and $F$ is possibly a set-valued mapping. The inclusion (10) covers a large territory including systems of equations and inequalities, variational inequalities, and equilibrium problems, as well as necessary optimality conditions in nonlinear programming and optimal control. Robinson's inverse function theorem is discussed in detail in [12, Chapter 2]. We only mention here the following version of it which is in the spirit of the Hildebrand-Graves theorem, Theorem 3, and is analogous to the extended Graves theorem, Theorem 2.

Theorem 4 (extended Hildebrand-Graves theorem). Consider a function $f$ : $X \rightarrow Y$ and a set-valued mapping $F: X \rightrightarrows Y$ along with positive constants $\kappa$ and $\mu$ such that $\kappa \mu<1$. Suppose that there exists a bounded linear mapping $A: X \rightarrow Y$ such that $\operatorname{lip}(f-A ; \bar{x}) \leq \mu$ and the mapping $f(\bar{x})+A(\cdot-\bar{x})+F(\cdot)$ is strongly metrically regular at $\bar{x}$ for $\bar{y}$ with $\operatorname{reg}(f(\bar{x})+A(\cdot-\bar{x})+F(\cdot) ; \bar{x} \mid \bar{y}) \leq \kappa$. Then the mapping $f+F$ is strongly metrically regular at $\bar{x}$ for $\bar{y}$; moreover,

$$
\operatorname{reg}(f+F ; \bar{x} \mid \bar{y}) \leq\left(\kappa^{-1}-\mu\right)^{-1} .
$$

The Hildebrand-Graves theorem, Theorem 3, is in sharp contrast with the classical (Dini) inverse function theorem in which differentiability plays a central role. In fact, the Hildebrand-Graves theorem is about nonsmooth functions, an area of analysis which emerged only in the 1970s. Among these developments is the inverse function theorem of Clarke [7], based on the generalized Jacobian introduced by him as a set-valued derivative-type approximation of a Lipschitz function. Recall that, according to a theorem by Rademacher, any function $f: \mathbb{R}^{n} \rightarrow \mathbb{R}^{d}$ which is Lipschitz continuous on an open set $O$ is differentiable almost everywhere in $O$. Clarke's generalized Jacobian of $f$ at $\bar{x} \in O$, denoted in this paper by $\bar{\partial} f(\bar{x})$, is the convex hull of all matrices obtained as limits of the usual Jacobians $\nabla f\left(x_{k}\right)$ for sequences $x_{k} \rightarrow \bar{x}$ such that $f$ is differentiable at $x_{k}$. Clarke's inverse function theorem says that for a function $f: \mathbb{R}^{n} \rightarrow \mathbb{R}^{n}$, which is Lipschitz continuous around $\bar{x}$ and such that every matrix in $\bar{\partial} f(\bar{x})$ is nonsingular, the inverse $f^{-1}$ has a Lipschitz continuous graphical localization around $f(\bar{x})$ for $\bar{x}$.

A Graves-type theorem utilizing Clarke's generalized Jacobian was obtained by Pourciau [26], who proved that a function $f: \mathbb{R}^{n} \rightarrow \mathbb{R}^{d}$, with $d \leq n$, which is Lipschitz continuous around $\bar{x}$, is metrically regular at $\bar{x}$ if every element of $\bar{\partial} f(\bar{x})$ has full rank. Note that Clarke's theorem provides only a sufficient condition for Lipschitz invertibility, and in the same way Pourciau's theorem gives a sufficient condition for metric regularity. Recently, Izmailov extended Clarke's theorem in [21, Theorem 1.3] to the framework of the inclusion (10) covering a finite-dimensional version of Robinson's theorem. A generalization of Izmailov's theorem to Banach spaces with a new proof is presented in the recent paper [4]; in section 4 of this paper we give a new proof of that generalization.

Observe that the Hildebrand-Graves theorem, Theorem 3, is quite different from Clarke's inverse function theorem, and the same is valid for the Graves theorem, Theorem 1, versus Pourciau's theorem. In Clarke's theorem the role of a derivative-type approximation is played by a set of matrices, which satisfies a certain condition. Páles [23] generalized both Pourciau's and Clarke's theorems to Banach spaces by utilizing Ioffe's strict prederivative [19]. Given a function $f: X \rightarrow Y$ and a point $\bar{x} \in \operatorname{int} \operatorname{dom} f$, the strict prederivative of $f$ at $\bar{x}$ is defined as a positively homogeneous mapping $\mathcal{A}: X \rightrightarrows Y$ with the following property: for every $\varepsilon>0$ there exists $\delta>0$ such that

$$
f\left(x^{\prime}\right) \in f(x)+\mathcal{A}\left(x^{\prime}-x\right)+\varepsilon\left\|x-x^{\prime}\right\| \mathbb{B} \quad \text { for every } \quad x^{\prime}, x \in \mathbb{B}_{\delta}(\bar{x}) .
$$


For our purposes it is more convenient to work with a subset $\mathcal{A}$ of $\mathcal{L}(X, Y)$ for which condition (11) holds. In finite dimensions Clarke's generalized Jacobian is an example of such a set. Further, to state his theorem, Páles also used the measure of non-compactness of $\mathcal{A}$, defined by

$$
\chi(\mathcal{A})=\inf \left\{r>0 \mid \mathcal{A} \subset \bigcup\left\{\mathbb{B}_{r}(A) \mid A \in \mathcal{B}\right\}, \mathcal{B} \subset \mathcal{A} \text { finite }\right\} .
$$

When $\mathcal{A}$ is represented by Clarke's generalized Jacobian this quantity is zero.

In his proof of the generalization of Pourciau's theorem in [23, Theorem 2] Páles used Michael's selection theorem, Ekeland's variational principle, and Kakutani's fixed point theorem. With minor updates in notation, Páles' theorem is as follows (with the convention that $0 \cdot \infty=\infty)$.

Theorem 5 (Páles (1997)). Let $f: X \rightarrow Y$ have a strict prederivative $\mathcal{A}$ at $\bar{x}$ which satisfies

$$
\chi(\mathcal{A}) \cdot \sup _{A \in \mathcal{A}}\left\|A^{-1}\right\|^{-}<1
$$

Then

$$
\operatorname{reg}(f ; \bar{x}) \leq\left(\left(\sup _{A \in \mathcal{A}}\left\|A^{-1}\right\|^{-}\right)^{-1}-\chi(\mathcal{A})\right)^{-1} .
$$

A generalization of Theorem 5 for the case when $f$ is defined only on a proper closed convex subset of $X$ rather than on the whole of $X$ is given in [6].

At the end of this introductory section we present a generalization of Theorem 2, a proof of which is given in section 2. Then we state our main result in Theorem 7, whose proof is given in section 3. Throughout, for given $\bar{x} \in X, \bar{y} \in Y$, a set $\mathcal{T} \subset \mathcal{L}(X, Y)$, and mappings $A \in \mathcal{T}, f: X \rightarrow Y$ and $F: X \rightarrow Y$, we utilize the mapping

$$
G_{A}: x \mapsto f(\bar{x})+A(x-\bar{x})+F(x)
$$

and denote

$$
\beta:=\sup _{A \in \mathcal{T}} \operatorname{reg}\left(G_{A} ; \bar{x} \mid \bar{y}\right) .
$$

TheOrem 6. Consider a function $f: X \rightarrow Y$, a set-valued mapping $F: X \rightarrow Y$, and a point $(\bar{x}, \bar{y}) \in \operatorname{gph}(f+F)$ with $\bar{x} \in \operatorname{int} \operatorname{dom} f$. Consider also a set $\mathcal{T}$ in $\mathcal{L}(X, Y)$ and a constant $\mu \geq 0$, and assume that the following conditions hold:

(A) there exists $r>0$ such that for each $u$ and $v$ in $\mathbb{B}_{r}(\bar{x})$ one can find $A \in \mathcal{T}$ with the following property:

$$
\|f(v)-f(u)-A(v-u)\| \leq \mu\|v-u\| ;
$$

(D) there exist neighborhoods $U$ of $\bar{x}, V$ of $\bar{y}$, and a positive real $\kappa$ such that for every $A \in \mathcal{T}$ the mapping $G_{A}^{-1}$, where $G_{A}$ is defined in (12), has the Aubin property at $\bar{y}$ for $\bar{x}$ with neighborhoods $U$ and $V$, and a constant $\kappa$. Furthermore, suppose that $\kappa, \mu$, and $\mathcal{T}$ satisfy

$$
\kappa(\mu+\operatorname{rad} \mathcal{T})<1
$$


Then the mapping $f+F$ is metrically regular at $\bar{x}$ for $\bar{y}$; moreover,

$$
\operatorname{reg}(f+F ; \bar{x} \mid \bar{y}) \leq\left(\kappa^{-1}-(\mu+\operatorname{rad} \mathcal{T})\right)^{-1} .
$$

Theorem 2 is a special case of Theorem 6 when $\mathcal{T}$ consists of one element only. A proof of this theorem is given in the next section.

Note that condition (D) requires the radii of the neighborhoods and the constant of the Aubin property of $G_{A}^{-1}$ be the same for all $A \in \mathcal{T}$, that is, the Aubin property is supposed to be uniform with respect to $A \in \mathcal{T}$. Another issue is the bound (15) involving the radius of the set $\mathcal{T}$ which may be hard to satisfy. Both these difficulties are taken care of in the following theorem, which is the main result of this paper.

THEOREM 7. Consider a function $f: X \rightarrow Y$, a set-valued mapping $F: X \rightrightarrows Y$, and a point $(\bar{x}, \bar{y}) \in \operatorname{gph}(f+F)$ with $\bar{x} \in \operatorname{int} \operatorname{dom} f$. Consider also a convex subset $\mathcal{T}$ of $\mathcal{L}(X, Y)$ and a constant $\mu \geq 0$, and assume that condition (A) stated in Theorem 6 as well as the following two conditions hold:

(B) for every $A \in \mathcal{T}$ the mapping $G_{A}$ defined in (12) is metrically regular at $\bar{x}$ for $\bar{y}$ and, in addition, for $\beta$ defined in (13),

$$
\beta(\mu+\chi(\mathcal{T}))<1
$$

(C) there are neighborhoods $U$ of $\bar{x}$ and $V$ of $\bar{y}$ such that the set $G_{A}^{-1}(v) \cap U$ is convex whenever $v \in V$ and $A \in \mathcal{T}$.

Then the mapping $f+F$ is metrically regular at $\bar{x}$ for $\bar{y}$; moreover,

$$
\operatorname{reg}(f+F ; \bar{x} \mid \bar{y}) \leq\left(B^{-1}-(\mu+\chi(\mathcal{T}))^{-1} .\right.
$$

Note the similarity in (15) and (16) but also the difference between these conditions when the set $\mathcal{T}$ is very large but compact. When $F$ is the zero mapping, Theorem 7 reduces to Páles' theorem, Theorem 5, if $\mathcal{T}$ is identified with the strict prederivative of $f$ at $\bar{x}$.

Both the Hildebrand-Graves theorem, Theorem 3, and the Graves theorem, Theorem 1, as well as, as a matter of fact, the Lyusternik theorem [22], were proved originally by using iterative procedures resembling the contraction mapping iteration. Theorem 2 is a special case of [12, Theorem 5E.1] for which several proofs are presented in Chapter 5 of that book. In a recent paper [5] Cibulka and Fabian obtained a result related to Theorem 7 but under different assumptions and with a different proof using Ioffe's criterion for regularity of mappings.

In section 2 we present first a proof of Theorem 6 and then some preparatory material for the proof of Theorem 7 -that proof is given in section 3 . In section 4, we show that the main results in [4] and [6] can be obtained as corollaries of Theorem 7 . In section 5 we consider the case when the function $f$ is represented by a Nemytskii operator and apply the abstract results obtained to derive a sufficient condition for metric regularity of a feasibility mapping in control.

2. A proof of Theorem 6 and preparation for proving Theorem 7 . We start this section with the following proof.

Proof of Theorem 6 . Let $a$ and $b$ be positive reals such that $\mathbb{B}_{a}(\bar{x}) \subset U$ and $\mathbb{B}_{b}(\bar{y}) \subset V$. Without loss of generality, suppose that the set $\operatorname{gph} G_{A} \cap\left(\mathbb{B}_{a}(\bar{x}) \times \mathbb{B}_{b}(\bar{y})\right)$ is closed for every $A \in \mathcal{T}$. Denote $\mu^{\prime}=\mu+\operatorname{rad} \mathcal{T}$ and let $\kappa^{\prime}>\left(\kappa^{-1}-\mu^{\prime}\right)^{-1}$. Choose $\delta>0$ such that

$$
\kappa\left(\mu^{\prime}+\delta\right)<1 \text { and } \kappa^{\prime}>\left(\kappa^{-1}-\left(\mu^{\prime}+\delta\right)\right)^{-1},
$$


and then find positive $\alpha$ and $\beta$ such that

$$
2 \kappa^{\prime} \beta+\alpha<\min \{a, r\} \text { and } \beta+\left(\mu^{\prime}+\delta\right)\left(2 \kappa^{\prime} \beta+\alpha\right)<b .
$$

Pick $A \in \mathcal{T}$ such that $\sup _{B \in \mathcal{T}}\|A-B\|<\operatorname{rad} \mathcal{T}+\delta$. We will show that for every $(u, y) \in \mathbb{B}_{2 \kappa^{\prime} \beta+\alpha}(\bar{x}) \times \mathbb{B}_{\beta}(\bar{y})$ one has

$$
y-f(u)+f(\bar{x})+A(u-\bar{x}) \in \mathbb{B}_{b}(\bar{y}) .
$$

Let $A^{\prime} \in \mathcal{T}$ be such that (14) holds with $v:=\bar{x}$. Then

$$
\begin{aligned}
\|y-f(u)+f(\bar{x})+A(u-\bar{x})-\bar{y}\| \leq & \|y-\bar{y}\|+\|f(\bar{x})-f(u)-A(\bar{x}-u)\| \\
\leq & \beta+\left\|f(\bar{x})-f(u)-A^{\prime}(\bar{x}-u)\right\| \\
& +\left\|\left(A^{\prime}-A\right)(\bar{x}-u)\right\| \\
\leq & \beta+\mu\|\bar{x}-u\|+(\operatorname{rad} \mathcal{T}+\delta)\|\bar{x}-u\| \\
\leq & \beta+\left(\mu^{\prime}+\delta\right)\left(2 \kappa^{\prime} \beta+\alpha\right)<b,
\end{aligned}
$$

where we use the second inequality in (18).

Fix any two distinct $y, y^{\prime} \in \mathbb{B}_{\beta}(\bar{y})$ and any $x^{\prime} \in(f+F)^{-1}\left(y^{\prime}\right) \cap \mathbb{B}_{\alpha}(\bar{x})$. Put $\varepsilon:=\kappa^{\prime}\left\|y-y^{\prime}\right\|$. Then $\varepsilon \leq 2 \kappa^{\prime} \beta$ and hence, from the first inequality in (18), we have

$$
\mathbb{B}_{\varepsilon}\left(x^{\prime}\right) \subset \mathbb{B}_{2 \kappa^{\prime} \beta+\alpha}(\bar{x}) \subset \mathbb{B}_{r}(\bar{x}) \cap \mathbb{B}_{a}(\bar{x}) .
$$

Define the mapping

$$
x \mapsto \Phi_{A}(x):=G_{A}^{-1}(y-f(x)+f(\bar{x})+A(x-\bar{x})) .
$$

By (19) both $w:=y-f\left(x^{\prime}\right)+f(\bar{x})+A\left(x^{\prime}-\bar{x}\right)$ and $w^{\prime}:=y^{\prime}-f\left(x^{\prime}\right)+f(\bar{x})+A\left(x^{\prime}-\bar{x}\right)$ are in $\mathbb{B}_{b}(\bar{y})$. Utilizing condition (D) and noting that $x^{\prime} \in G_{A}^{-1}\left(w^{\prime}\right) \cap \mathbb{B}_{a}(\bar{x})$, we get

$$
\begin{aligned}
d\left(x^{\prime}, \Phi_{A}\left(x^{\prime}\right)\right) & =d\left(x^{\prime}, G_{A}^{-1}(w)\right) \leq e\left(G_{A}^{-1}\left(w^{\prime}\right) \cap \mathbb{B}_{a}(\bar{x}), G_{A}^{-1}(w)\right) \leq \kappa\left\|w-w^{\prime}\right\| \\
& =\kappa\left\|y-y^{\prime}\right\|<\kappa^{\prime}\left\|y-y^{\prime}\right\|\left(1-\kappa\left(\mu^{\prime}+\delta\right)\right)=\varepsilon\left(1-\kappa\left(\mu^{\prime}+\delta\right)\right) .
\end{aligned}
$$

Let $u, v \in \mathbb{B}_{\varepsilon}\left(x^{\prime}\right)$. By (19) both $w_{u}:=y-f(u)+f(\bar{x})+A(u-\bar{x})$ and $w_{v}:=$ $y-f(v)+f(\bar{x})+A(v-\bar{x})$ are in $\mathbb{B}_{b}(\bar{y})$. Now, let $\bar{A}$ be associated with $u$ and $v$ according to condition (A). Then condition (D) gives us

$$
\begin{aligned}
e\left(\Phi_{A}(u) \cap \mathbb{B}_{\varepsilon}\left(x^{\prime}\right), \Phi_{A}(v)\right) & =e\left(G_{A}^{-1}\left(w_{u}\right) \cap \mathbb{B}_{\varepsilon}\left(x^{\prime}\right), G_{A}^{-1}\left(w_{v}\right)\right) \\
& \leq e\left(G_{A}^{-1}\left(w_{u}\right) \cap \mathbb{B}_{a}(\bar{x}), G_{A}^{-1}\left(w_{v}\right)\right) \leq \kappa\left\|w_{u}-w_{v}\right\| \\
& \leq \kappa(\|f(v)-f(u)-\bar{A}(v-u)\|+\|(\bar{A}-A)(v-u)\|) \\
& \leq \kappa(\mu+\operatorname{rad} \mathcal{T}+\delta)\|v-u\|=\kappa\left(\mu^{\prime}+\delta\right)\|u-v\| .
\end{aligned}
$$

We need to also show that the set $\mathcal{F}:=\operatorname{gph} \Phi_{A} \cap\left(\mathbb{B}_{\varepsilon}\left(x^{\prime}\right) \times \mathbb{B}_{\varepsilon}\left(x^{\prime}\right)\right)$ is closed. Let $\left(x_{n}, z_{n}\right)$ be a sequence in $\mathcal{F}$ which converges to $(\tilde{x}, \tilde{z})$. Then clearly $(\tilde{x}, \tilde{z}) \in \mathbb{B}_{\varepsilon}\left(x^{\prime}\right) \times \mathbb{B}_{\varepsilon}\left(x^{\prime}\right)$. Furthermore, by (19) we have

$$
\begin{aligned}
\left(z_{n}, y-f\left(x_{n}\right)+f(\bar{x})+A\left(x_{n}-\bar{x}\right)\right) & \in \operatorname{gph} G_{A} \cap\left(\mathbb{B}_{\varepsilon}\left(x^{\prime}\right) \times \mathbb{B}_{b}(\bar{y})\right) \\
& \subset \operatorname{gph} G_{A} \cap\left(\mathbb{B}_{a}(\bar{x}) \times \mathbb{B}_{b}(\bar{y})\right) \text { for each } n .
\end{aligned}
$$

Passing to the limit we get that $(\tilde{z}, y-f(\tilde{x})+f(\bar{x})+A(\tilde{x}-\bar{x})) \in \operatorname{gph} G_{A}$, that is, $(\tilde{x}, \tilde{z}) \in \operatorname{gph} \Phi_{A}$, which completes the proof of the closedness of $\mathcal{F}$. 
We can now apply the contraction mapping theorem proved in [10] (see also [12, Theorem 5E.2]) to obtain that there exists a fixed point $x \in \Phi_{A}(x) \cap \mathbb{B}_{\varepsilon}\left(x^{\prime}\right)$, that is, $x \in(f+F)^{-1}(y)$ with $\left\|x-x^{\prime}\right\| \leq \kappa^{\prime}\left\|y-y^{\prime}\right\|$. This means that $(f+F)^{-1}$ has the Aubin property at $\bar{y}$ for $\bar{x}$ with constant $\kappa^{\prime}$, hence $f+F$ is metrically regular at $\bar{x}$ for $\bar{y}$ with constant $\kappa^{\prime}$.

The proof of Theorem 7 presented in the next section uses extended versions of the theorem of Graves stated in [12, Theorem 5G.3] and [12, Theorem 5E.5]. Specifically, in Lemma 12 we prove that the mapping $G_{A}^{-1}$ has the Aubin property with the same constant and neighborhoods for all $A \in \mathcal{B}$, where $\mathcal{B}$ is a compact convex subset of $\mathcal{A}$. Then in Lemma 13 we apply Michael's selection theorem to a mapping $H$ defined as the composition of $G_{A}^{-1}$ and the "nonlinear part" of $f$. By applying Gliksberg's extension of Kakutani's fixed point theorem, in Lemma 14 we show that a composition of certain mapping with this selection has a fixed point. Then in the last part of the proof we show that the mapping $(f+F)^{-1}$ has the Aubin property, by constructing a sequence of points $x_{n}$ and operators $A_{n} \in \mathcal{B}$ that converge to a limit which gives us the desired property.

We present next some auxiliary results used in the proof of Theorem 7. In that proof we utilize the property of metric regularity on a set. Given nonempty sets $U \subset X$ and $V \subset Y$ and a constant $\kappa \geq 0$, a set-valued mapping $\Phi: X \rightrightarrows Y$ is said to be metrically regular on $U$ for $V$ with constant $\kappa$ when the set gph $\Phi \cap(U \times V)$ is closed and

$$
d\left(x, \Phi^{-1}(y)\right) \leq \kappa d(y, \Phi(x) \cap V) \quad \text { for all } \quad(x, y) \in U \times V .
$$

The link between the properties of metric regularity on sets and at points is given by the following proposition.

Proposition 8 (see [12, Proposition 5H.1]). For positive scalars $a, b$, and $\kappa$ and a point $(\bar{x}, \bar{y}) \in X \times Y$ consider a mapping $\Phi: X \rightarrow Y$ with $\bar{y} \in \Phi(\bar{x})$ which is metrically regular on $\mathbb{B}_{a}(\bar{x})$ for $\mathbb{B}_{b}(\bar{y})$ with constant $\kappa$. Then $\Phi$ is metrically regular at $\bar{x}$ for $\bar{y}$ with constant $\kappa$.

The following theorem is a part of [12, Theorem 5G.3] and concerns perturbed metric regularity.

TheOrem 9. Let $a, b$, and $\kappa$ be positive scalars such that $F$ is metrically regular at $\bar{x}$ for $\bar{y}$ with neighborhoods $\mathbb{B}_{a}(\bar{x})$ and $\mathbb{B}_{b}(\bar{y})$ and constant $\kappa$. Let $L>0$ be such that $\kappa L<1$ and let $\kappa^{\prime}>\kappa /(1-\kappa L)$. Then for every positive $\alpha$ and $\beta$ such that

$$
\alpha \leq a / 2, \quad 2 L \alpha+2 \beta \leq b \quad \text { and } \quad 2 \kappa^{\prime} \beta \leq \alpha
$$

and for every function $g: X \rightarrow Y$ satisfying

$$
\|g(\bar{x})\| \leq \beta \quad \text { and } \quad\left\|g(x)-g\left(x^{\prime}\right)\right\| \leq L\left\|x-x^{\prime}\right\| \quad \text { for every } x, x^{\prime} \in \mathbb{B}_{2 \alpha}(\bar{x}),
$$

the mapping $g+F$ has the following property: for every $y, y^{\prime} \in \mathbb{B}_{\beta}(\bar{y})$ and every $x \in(g+F)^{-1}(y) \cap \mathbb{B}_{\alpha}(\bar{x})$ there exists $x^{\prime} \in(g+F)^{-1}\left(y^{\prime}\right)$ such that

$$
\left\|x-x^{\prime}\right\| \leq \kappa^{\prime}\left\|y-y^{\prime}\right\| .
$$

In the original statement of [12, Theorem 5G.3] it is assumed that in (21) one has $L \alpha+2 \beta \leq b$ and that the Lipschitz estimate in (22) holds for all $x, x^{\prime} \in \mathbb{B}_{\alpha}(\bar{x})$. 
It turns out that there is a glitch in the proof ${ }^{1}$ which can be easily fixed: $\alpha$ should be replaced by $2 \alpha$ and then in the proof one has $\mathbb{B}_{r}(x) \subset \mathbb{B}_{2 \alpha}(\bar{x}) \subset \mathbb{B}_{a}(\bar{x})$, where $r:=\kappa^{\prime}\left\|y-y^{\prime}\right\|$.

In the proof of Theorem 7 we will also employ the following corollary of $[12$, Theorem 5E.5].

Theorem 10. Let $X, Y$, and $P$ be Banach spaces, and let $g: P \times X \rightarrow Y$ be a function defined on a neighborhood of a point $(\bar{p}, \bar{x}) \in P \times X$ such that $g(\bar{p}, \bar{x})=0$. For a mapping $\Phi: X \rightrightarrows Y$ with $\Phi(\bar{x}) \ni 0$ consider the generalized equation $g(p, x)+\Phi(x) \ni$ 0 with the associated solution mapping

$$
P \ni p \mapsto S(p)=\{x \in X \mid g(p, x)+\Phi(x) \ni 0\} .
$$

Suppose that

(i) there is a constant $\nu>0$ along with neighborhoods $Q$ of $\bar{p}$ and $U$ of $\bar{x}$ such that

$$
\left\|g(p, x)-g\left(p, x^{\prime}\right)\right\| \leq \nu\left\|x-x^{\prime}\right\| \quad \text { whenever } \quad(p, x),\left(p, x^{\prime}\right) \in Q \times U ;
$$

(ii) there is a constant $\gamma>0$ along with neighborhoods $Q^{\prime}$ of $\bar{p}$ and $U^{\prime}$ of $\bar{x}$ such that

$$
\left\|g(p, x)-g\left(p^{\prime}, x\right)\right\| \leq \gamma\left\|p-p^{\prime}\right\| \quad \text { whenever } \quad(p, x),\left(p^{\prime}, x\right) \in Q^{\prime} \times U^{\prime} ;
$$

(iii) $\Phi$ is metrically regular at $\bar{x}$ for 0 with $\operatorname{reg}(\Phi ; \bar{x} \mid 0)<\kappa<1 / \nu$.

Then there are neighborhoods $Q^{\prime \prime}$ of $\bar{p}$ and $U^{\prime \prime}$ of $\bar{x}$ such that

$$
S(p) \cap U^{\prime \prime} \subset S\left(p^{\prime}\right)+\frac{\kappa \gamma}{1-\kappa \nu}\left\|p-p^{\prime}\right\| \mathbb{B} \quad \text { for every } p, p^{\prime} \in Q^{\prime \prime} .
$$

Finally, in the proof of Theorem 7 we utilize the following observation which we state as a lemma.

Lemma 11. Let $T: X \rightrightarrows Y, v \in Y$, and $r>0$ be such that the mapping $\Phi_{1}$ : $x \mapsto T(x) \cap \stackrel{\mathrm{o}}{\mathbb{B}}_{r}(v)$ is inner semicontinuous in its domain and the mapping $\Phi_{2}: x \mapsto$ $T(x) \cap \mathbb{B}_{r}(v)$ is convex-valued. Then $\Phi_{2}$ is inner semicontinuous on $\operatorname{dom} \Phi_{1}$.

Proof. Let $x_{0} \in \operatorname{dom} \Phi_{1}$ and $y_{0} \in \Phi_{2}\left(x_{0}\right)$, and let $V$ be an open neighborhood of $y_{0}$ in $Y$. First, let $\left\|y_{0}-v\right\|<r$. The inner semicontinuity of $\Phi_{1}$ yields the existence of an open neighborhood $U$ of $x_{0}$ such that $\emptyset \neq T(x) \cap \stackrel{\circ}{\mathbb{B}_{r}}(v) \cap V \subset \Phi_{2}(x) \cap V$ for all $x \in U$. Now, let $\left\|y_{0}-v\right\|=r$. Pick any $\hat{y} \in T\left(x_{0}\right) \cap \stackrel{\mathrm{o}}{\mathbb{B}}_{r}(v)$. Since the set $T\left(x_{0}\right) \cap \mathbb{B}_{r}(v)$ is convex and contains both $\hat{y}$ and $y_{0}$ there exists $\tilde{y} \in T\left(x_{0}\right) \cap \stackrel{\mathrm{o}}{\mathbb{B}}_{r}(v) \cap V$. Hence again there exists an open neighborhood $U$ of $x_{0}$ such that $\emptyset \neq T(x) \cap \mathbb{B}_{r}(v) \cap V \subset \Phi_{2}(x) \cap V$ for all $x \in U$ and we are done.

3. Proof of Theorem 7. Without loss of generality, let $\bar{y}=0$. Let $r>0$ and $\beta$ be as in the statement of the theorem. By assumption (B), one can choose positive constants $\varepsilon$ and $\ell$ such that

$$
\varepsilon>\mu+\chi(\mathcal{T}), \quad \ell>\beta \quad \text { and } \quad \ell \varepsilon<1
$$

\footnotetext{
${ }^{1}$ Many thanks to Jakob Preininger from Technical University of Vienna who discovered this mistake.
} 
By the definition of the measure of noncompactness $\chi(\mathcal{T})$, there exists a finite set $\mathcal{A} \subset \mathcal{L}(X, Y)$ such that

$$
\mathcal{A} \subset \mathcal{T} \subset \mathcal{A}+(\varepsilon-\mu) \mathbb{B} .
$$

Denote by $\mathcal{B}$ the convex hull of $\mathcal{A}$. Since $\mathcal{A}$ is finite and $\mathcal{T}$ is convex, the set $\mathcal{B}$ is a compact convex subset of $\mathcal{T}$. Choose $\beta^{\prime}$ such that $\ell>\beta^{\prime}>\beta$ and let $\gamma>0$ satisfy

$$
\gamma \beta^{\prime}<1 \quad \text { and } \quad \frac{\beta^{\prime}}{1-\gamma \beta^{\prime}}<\ell-\gamma .
$$

Our first lemma shows that under the current assumptions, the Aubin property of the mapping $G_{A}^{-1}$ is actually uniform in $A \in \mathcal{B}$, a property we required in Theorem 6 .

Lemma 12. There exists $\beta>0$ such that for every $A \in \mathcal{B}$ the mapping $G_{A}$ defined in (12) has the following property: for every $v, v^{\prime} \in \mathbb{B}_{\beta}(0)$ and every $u \in$ $G_{A}^{-1}(v) \cap \mathbb{B}_{2 \ell \beta}(\bar{x})$ there exists $u^{\prime} \in G_{A}^{-1}\left(v^{\prime}\right)$ such that

$$
\left\|u^{\prime}-u\right\| \leq(\ell-\gamma)\left\|v^{\prime}-v\right\| .
$$

Proof. We show first that for each $\bar{A} \in \mathcal{B}$ there is $\beta_{\bar{A}}>0$ such that for each $A \in \mathbb{B}_{\gamma}(\bar{A})$ one has that for every $v, v^{\prime} \in \mathbb{B}_{\beta_{\bar{A}}}(0)$ and every $u \in G_{A}^{-1}(v) \cap \mathbb{B}_{2 \ell \beta_{\bar{A}}}(\bar{x})$ there exists $u^{\prime} \in G_{A}^{-1}\left(v^{\prime}\right)$ such that

$$
\left\|u^{\prime}-u\right\| \leq(\ell-\gamma)\left\|v^{\prime}-v\right\|
$$

Choose any $\bar{A} \in \mathcal{B}$. By the assumed metric regularity of $G_{\bar{A}}$ in (B), there exist $a>0$ and $b>0$ (depending on $\bar{A}$ ) such that $G_{\bar{A}}$ is metrically regular at $\bar{x}$ for 0 with neighborhoods $\mathbb{B}_{a}(\bar{x})$ and $\mathbb{B}_{b}(0)$ and constant $\beta^{\prime}$. Pick any $A \in \mathbb{B}_{\gamma}(\bar{A})$ and define the function

$$
g(u):=(A-\bar{A})(u-\bar{x}), \quad u \in X .
$$

We have $G_{A}=G_{\bar{A}}+g, g(\bar{x})=0$, and also

$$
\left\|g(x)-g\left(x^{\prime}\right)\right\|=\left\|(A-\bar{A})\left(x-x^{\prime}\right)\right\| \leq \gamma\left\|x-x^{\prime}\right\| \quad \text { for any } x, x^{\prime} \in X .
$$

We apply Theorem 9 with $F=G_{\bar{A}}, \bar{y}=0, \kappa:=\beta^{\prime}, \kappa^{\prime}:=\ell-\gamma$, and $L:=\gamma$. From (24) we get

$$
\kappa L=B^{\prime} \gamma<1 \quad \text { and } \kappa^{\prime}=\ell-\gamma>B^{\prime} /\left(1-B^{\prime} \gamma\right)=\kappa /(1-\kappa L) .
$$

Moreover, (22) is fulfilled for any $\alpha>0$ and $\beta>0$. Hence, the inequalities in (21) hold when one takes

$$
\beta=\beta_{\bar{A}}:=\min \left\{\frac{a}{4 \ell}, \frac{b}{2(2 \gamma \ell+1)}\right\}, \quad \alpha=\alpha_{\bar{A}}:=2 \ell \beta_{\bar{A}} .
$$

Then Theorem 9 implies the desired property of the mapping $G_{A}=G_{\bar{A}}+g$.

Since $\mathcal{B}$ is compact, from the open covering $\bigcup_{A \in \mathcal{B}} \mathbb{B}_{\gamma}(A)$ of $\mathcal{B}$ we can choose a finite subcovering with open balls $\stackrel{\mathrm{o}}{\mathbb{B}}_{\gamma}\left(\bar{A}_{i}\right)$ for some subset $\left\{\bar{A}_{1}, \ldots, \bar{A}_{k}\right\}$ of $\mathcal{B}$, say, with cardinality $k$. Taking the corresponding $\beta_{\bar{A}_{i}}>0$ for each $i \in\{1, \ldots, k\}$, then $\beta:=\min _{i \in\{1, \ldots, k\}} \beta_{\bar{A}_{i}}$ is the desired quantity. 
Continuing with the proof, from condition (A) and the inclusion $\mathcal{T} \subset \mathcal{A}+(\varepsilon-\mu) \mathbb{B}$ we obtain

$$
\begin{gathered}
\text { for every } u, v \in \mathbb{B}_{r}(\bar{x}) \text { there is } A \in \mathcal{B} \text { such that } \\
\|f(v)-f(u)-A(v-u)\| \leq \varepsilon\|v-u\| .
\end{gathered}
$$

Let $c:=\sup _{A \in \mathcal{B}}\|A\| ;$ then, from (25),

$$
\|f(v)-f(u)\| \leq(c+\varepsilon)\|v-u\| \quad \text { for every } u, v \in \mathbb{B}_{r}(\bar{x}),
$$

that is, $f$ is Lipschitz continuous on $\mathbb{B}_{r}(\bar{x})$ with a Lipschitz constant $c+\varepsilon$. Clearly, in Lemma 12 we can make $\beta$ smaller without changing anything; let $\beta>0$ be such that

$$
\mathbb{B}_{2 \ell \beta}(\bar{x}) \times \mathbb{B}_{\beta}(0) \subset U \times V,
$$

where $U$ and $V$ are the neighborhoods in condition (C), and also

$$
\text { the set }\left(\mathbb{B}_{2 \ell \beta}(\bar{x}) \times \mathbb{B}_{\beta}(-f(\bar{x}))\right) \cap \operatorname{gph} F \text { is closed. }
$$

That the latter is possible comes from the assumed metric regularity in (B) according to which the graph of each $G_{A}$ is locally closed at $(\bar{x}, 0)$, hence gph $F$ is locally closed at $(\bar{x},-f(\bar{x}))$. Pick $\delta \in(0, r / 7)$ such that

$$
6 \delta<\frac{\beta}{(1 / \ell+3 c)} .
$$

Clearly, $4 \delta<\ell \beta$. From (23),

$$
b:=(1-\varepsilon \ell) \delta<\delta
$$

For any $y \in \mathbb{B}_{3 \varepsilon b}(0), w \in \mathbb{B}_{3 \delta}(\bar{x}), \tilde{u} \in \mathbb{B}_{8 \delta}(\bar{x})$, and $A \in \mathcal{B}$ the relations (27) and (28) yield that

$$
\begin{aligned}
\|y-f(w)+f(\bar{x})+A(w-\tilde{u})\| & \leq\|y\|+\|f(w)-f(\bar{x})\|+\|A(w-\bar{x})\|+\|A(\tilde{u}-\bar{x})\| \\
& \leq 3 \varepsilon b+(\varepsilon+c)\|w-\bar{x}\|+c\|w-\bar{x}\|+c\|\tilde{u}-\bar{x}\| \\
& \leq 3 \varepsilon b+(\varepsilon+c) 3 \delta+11 c \delta<\delta(6 \varepsilon+14 c)<6 \delta(1 / \ell+3 c) \\
& <\beta .
\end{aligned}
$$

Hence, for each $(y, w, \tilde{u}, A) \in \mathbb{B}_{3 \varepsilon b}(0) \times \mathbb{B}_{3 \delta}(\bar{x}) \times \mathbb{B}_{8 \delta}(\bar{x}) \times \mathcal{B}$ we have

$$
y-f(w)+f(\bar{x})+A(w-\tilde{u}) \in \mathbb{B}_{\beta}(0) .
$$

The next step of the proof is the following lemma.

Lemma 13. For every $x \in \mathbb{B}_{3 \delta}(\bar{x})$, every $y \in \mathbb{B}_{\varepsilon b}(0)$, and every $y^{\prime} \in \mathbb{B}_{3 \varepsilon b}(0)$ such that $x \in(f+F)^{-1}\left(y^{\prime}\right)$ the mapping

$$
\mathcal{B} \ni A \mapsto H(A):=G_{A}^{-1}(y-f(x)+f(\bar{x})+A(x-\bar{x})) \cap \mathbb{B}_{\ell\left\|y-y^{\prime}\right\|}(x)
$$

has a continuous selection on $\mathcal{B}$.

Proof. If $y=y^{\prime}$, then the claim holds trivially since $H(A)=\{x\}$ for any $A \in \mathcal{B}$. Assume that $y \neq y^{\prime}$ and along with $H$ consider the mapping

$$
\mathcal{B} \ni A \mapsto \widetilde{H}(A):=G_{A}^{-1}(y-f(x)+f(\bar{x})+A(x-\bar{x})) \cap \stackrel{\circ}{\mathbb{B}_{\ell\left\|y-y^{\prime}\right\|}}(x) .
$$


We will show first that $H$ has closed convex values and $\operatorname{dom} H=\operatorname{dom} \widetilde{H}=\mathcal{B}$. Choose any $A \in \mathcal{B}$. Let

$$
v:=y^{\prime}-f(x)+f(\bar{x})+A(x-\bar{x}) \quad \text { and } \quad v^{\prime}:=y-f(x)+f(\bar{x})+A(x-\bar{x}) .
$$

Utilizing (29) we obtain $v, v^{\prime} \in \mathbb{B}_{\beta}(0)$. Since

$$
\mathcal{B} \subset \mathcal{T} \text { and } \mathbb{B}_{\ell\left\|y-y^{\prime}\right\|}(x) \subset \mathbb{B}_{4 \ell \varepsilon b+3 \delta}(\bar{x}) \subset \mathbb{B}_{8 \delta}(\bar{x}) \subset \mathbb{B}_{2 \beta \ell}(\bar{x}),
$$

condition (C) together with (26) implies that the set $H(A)=G_{A}^{-1}\left(v^{\prime}\right) \cap \mathbb{B}_{\ell\left\|y-y^{\prime}\right\|}(x)$ is convex. Note that, by (27), $3 \delta<\ell \beta$; hence $x \in G_{A}^{-1}(v) \cap \mathbb{B}_{\ell \beta}(\bar{x})$. Applying Lemma 12 with $u:=x$, we obtain that there exists $u \in G_{A}^{-1}\left(v^{\prime}\right)$ such that $\|u-x\| \leq(\ell-\gamma)\left\|y-y^{\prime}\right\|$, that is,

$$
u \in G_{A}^{-1}(y-f(x)+f(\bar{x})+A(x-\bar{x})) \cap \stackrel{\circ}{\mathbb{B}}_{\ell\left\|y-y^{\prime}\right\|}(x)=\widetilde{H}(A) \subset H(A) .
$$

To prove that the set $H(A)$ is closed, let $\left\{u_{n}\right\}$ be any sequence in $H(A)$ converging to $u \in X$. Then, by (29), for each natural $n$ we have

$$
\begin{aligned}
\left(u_{n}, y-f(x)-A\left(u_{n}-x\right)\right) & \in\left(\mathbb{B}_{\ell\left\|y-y^{\prime}\right\|}(x) \times \mathbb{B}_{\beta}(-f(\bar{x}))\right) \cap \operatorname{gph} F \\
& \subset\left(\mathbb{B}_{2 \beta \ell}(\bar{x}) \times \mathbb{B}_{\beta}(-f(\bar{x}))\right) \cap \operatorname{gph} F .
\end{aligned}
$$

Since in the last displayed formula the set on the right is closed, we conclude that

$$
(u, y-f(x)-A(u-x)) \in\left(\mathbb{B}_{\ell\left\|y-y^{\prime}\right\|}(x) \times \mathbb{B}_{\beta}(-f(\bar{x}))\right) \cap \operatorname{gph} F .
$$

Thus $u \in H(A)$.

We show next that $H$ is inner semicontinuous on $\mathcal{B}$. In view of Lemma 11 it is sufficient to show that the mapping $\widetilde{H}$ is inner semicontinuous on $\mathcal{B}$. Let $\bar{A} \in \mathcal{B}$, let $\bar{u} \in \widetilde{H}(\bar{A})$, and define the mappings

$$
\Phi(u):=f(x)-y+\bar{A}(u-x)+F(u), \quad u \in X,
$$

and

$$
g(A, u):=(A-\bar{A})(u-x), \quad(A, u) \in \mathcal{L}(X, Y) \times X .
$$

Then

$$
\Phi(\bar{u}) \ni 0 \quad \text { and } \quad g(\bar{A}, \bar{u})=0 .
$$

Choose a positive $\nu$ such that $\nu \ell<1$. Then for every choice of $A \in \mathbb{B}_{\nu}(\bar{A})$ and $u$, $u^{\prime} \in X$ we have

$$
\left\|g(A, u)-g\left(A, u^{\prime}\right)\right\| \leq\|A-\bar{A}\|\left\|u-u^{\prime}\right\| \leq \nu\left\|u-u^{\prime}\right\| .
$$

Moreover, for every $A, A^{\prime} \in \mathcal{L}(X, Y)$ and every $u \in \mathbb{B}_{3 \delta}(\bar{x})$, we get

$$
\left\|g(A, u)-g\left(A^{\prime}, u\right)\right\| \leq\left\|A-A^{\prime}\right\|\|u-x\| \leq 6 \delta\left\|A-A^{\prime}\right\| .
$$

Let us now show that $\Phi$ is metrically regular at $\bar{u}$ for 0 with constant $\ell-\gamma$. In view of Proposition 8, it suffices to prove that

$$
d\left(u, \Phi^{-1}(w)\right) \leq(\ell-\gamma) d\left(w, \Phi(u) \cap \mathbb{B}_{\varepsilon b}(0)\right) \quad \text { for all } \quad(u, w) \in \mathbb{B}_{\delta}(\bar{u}) \times \mathbb{B}_{\varepsilon b}(0)
$$


and that $\left(\mathbb{B}_{\delta}(\bar{u}) \times \mathbb{B}_{\varepsilon b}(0)\right) \cap \operatorname{gph} \Phi$ is closed. Since $\|\bar{u}-x\|<\ell\left\|y-y^{\prime}\right\| \leq 4 \varepsilon \ell b<4 b<4 \delta$, we have $\|\bar{u}-\bar{x}\|<4 \delta+3 \delta=7 \delta$. Hence, taking into account that $4 \delta<\beta \ell$, we get

$$
\mathbb{B}_{\delta}(\bar{u}) \times \mathbb{B}_{\varepsilon b}(0) \subset \mathbb{B}_{8 \delta}(\bar{x}) \times \mathbb{B}_{\delta / \ell}(0) \subset \mathbb{B}_{2 \ell \beta}(\bar{x}) \times \mathbb{B}_{\beta / 4}(0) .
$$

Note that $(u, w) \in \operatorname{gph} \Phi$ if and only if $(u, w+y-f(x)+\bar{A}(x-u)) \in \operatorname{gph} F$. Moreover, if $(u, w) \in \mathbb{B}_{\delta}(\bar{u}) \times \mathbb{B}_{\varepsilon b}(0)$, then the combination of (32) and (29) with $y:=w+y$, $w:=x, \tilde{u}:=u$, and $A=\bar{A}$ implies that

$$
(u, w+y-f(x)+\bar{A}(x-u)) \in \mathbb{B}_{2 \ell \beta}(\bar{x}) \times \mathbb{B}_{\beta}(-f(\bar{x})) .
$$

Since the set $\left(\mathbb{B}_{2 \ell \beta}(\bar{x}) \times \mathbb{B}_{\beta}(-f(\bar{x}))\right) \cap \operatorname{gph} F$ is closed, so is $\left(\mathbb{B}_{\delta}(\bar{u}) \times \mathbb{B}_{\varepsilon b}(0)\right) \cap \operatorname{gph} \Phi$.

Fix $(u, w) \in \mathbb{B}_{\delta}(\bar{u}) \times \mathbb{B}_{\varepsilon b}(0)$. If $\Phi(u) \cap \mathbb{B}_{\varepsilon b}(0)=\emptyset$, then (31) holds automatically. If not, pick $w^{\prime} \in \Phi(u) \cap \mathbb{B}_{\varepsilon b}(0)$. Let

$$
v:=w^{\prime}+y-f(x)+f(\bar{x})+\bar{A}(x-\bar{x}) \quad \text { and } \quad v^{\prime}:=w+y-f(x)+f(\bar{x})+\bar{A}(x-\bar{x}) .
$$

By (29) with $w:=x, \tilde{u}:=\bar{x}, A:=\bar{A}$, and $y$ replaced by $w+y$ and $w^{\prime}+y$, respectively, we have $v, v^{\prime} \in \mathbb{B}_{\beta}(0)$. Since $w^{\prime} \in \Phi(u)$, we obtain $u \in G_{\bar{A}}^{-1}(v)$. Moreover, (32) implies that $u \in \mathbb{B}_{2 \ell \beta}(\bar{x})$. Lemma 12 then can be applied, yielding the existence of $u^{\prime} \in G_{\bar{A}}^{-1}\left(v^{\prime}\right)$ such that

$$
\left\|u-u^{\prime}\right\| \leq(\ell-\gamma)\left\|v-v^{\prime}\right\|=(\ell-\gamma)\left\|w-w^{\prime}\right\| .
$$

Then $w \in \Phi\left(u^{\prime}\right)$ and thus

$$
d\left(u, \Phi^{-1}(w)\right) \leq\left\|u-u^{\prime}\right\| \leq(\ell-\gamma)\left\|w-w^{\prime}\right\| .
$$

Since $w^{\prime} \in \Phi(u) \cap \mathbb{B}_{\varepsilon b}(0)$ was arbitrarily chosen, we get (31). Hence, $\Phi$ is metrically regular at $\bar{u}$ for 0 with constant $\ell-\gamma$.

We can now apply Theorem 10 with $P:=\mathcal{L}(X, Y), \kappa:=\ell$, and $\gamma:=6 \delta$ obtaining that there exists $\gamma^{\prime}>0$ such that for each $A \in \mathcal{B}$ with $\|A-\bar{A}\|<\gamma^{\prime}$ there is $u(A) \in X$ satisfying

$$
g(A, u(A))+\Phi(u(A)) \ni 0 \quad \text { and } \quad\|u(A)-\bar{u}\| \leq \frac{6 \delta \ell}{1-\nu \ell}\|A-\bar{A}\| .
$$

Note that $g(A, u)+\Phi(u)=G_{A}(u)-y+f(x)-f(\bar{x})-A(x-\bar{x})$ for any $(u, A) \in$ $X \times \mathcal{L}(X, Y)$. Since $\|\bar{u}-x\|<\ell\left\|y-y^{\prime}\right\|$, making $\gamma^{\prime}$ smaller if necessary, we obtain

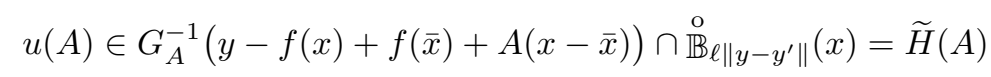

whenever $\|A-\bar{A}\|<\gamma^{\prime}$. This proves the inner semicontinuity of the mapping $\widetilde{H}$ at $\bar{A}$ which was chosen arbitrarily in $\mathcal{B}$; thus, $\widetilde{H}$ is inner semicontinuous on $\mathcal{B}$, and hence so is $H$.

We showed that the mapping $H$ is inner semicontinuous and has nonempty closed convex values on $\mathcal{B}$. Michael's selection theorem (see, e.g., [14]) yields the existence of the desired continuous selection.

Choose any $x \in \mathbb{B}_{3 \delta}(\bar{x}), y \in \mathbb{B}_{\varepsilon b}(0)$, and $y^{\prime} \in \mathbb{B}_{3 \varepsilon b}(0)$ such that $x \in(f+F)^{-1}\left(y^{\prime}\right)$. From Lemma 13 we obtain that the mapping $\mathcal{B} \ni A \mapsto H(A)-x$, where $H$ is as in (30), has a continuous selection in $\mathcal{B}$. Denote this selection by $\varphi_{x, y, y^{\prime}}$. Keep $x \in \mathbb{B}_{3 \delta}(\bar{x})$ fixed and define the following set-valued mapping acting from $X$ into the subsets of $\mathcal{B}$ :

$$
X \ni h \mapsto \Psi_{x}(h):=\{A \in \mathcal{B} \mid\|f(x+h)-f(x)-A h\| \leq \varepsilon\|h\|\} .
$$


LEMma 14. Given $x \in \mathbb{B}_{3 \delta}(\bar{x}), y \in \mathbb{B}_{\varepsilon b}(0)$, and $y^{\prime} \in \mathbb{B}_{3 \varepsilon b}(0)$ such that $x \in$ $(f+F)^{-1}\left(y^{\prime}\right)$, the composition mapping $\Psi_{x} \circ \varphi_{x, y, y^{\prime}}$ acting from $\mathcal{B}$ into itself has a fixed point.

Proof. Since $f$ is continuous, the mapping $\Psi_{x}$ has closed graph. Note that $\varphi_{x, y, y^{\prime}}(\mathcal{B}) \subset \operatorname{dom} \Psi_{x}$. Indeed, fix any $A \in \mathcal{B}$. Then there exists $\tilde{x} \in \mathbb{B}_{\ell\left\|y-y^{\prime}\right\|}(x)$ such that $\varphi_{x, y, y^{\prime}}(A)=\tilde{x}-x$. Hence $\left\|\varphi_{x, y, y^{\prime}}(A)\right\| \leq \ell\left\|y-y^{\prime}\right\|$ and therefore

$$
\left\|x+\varphi_{x, y, y^{\prime}}(A)-\bar{x}\right\|=\|x-\bar{x}\|+\ell\left\|y-y^{\prime}\right\| \leq 3 \delta+4 \varepsilon \ell b<7 \delta<r .
$$

Then (25) with $v:=x+\varphi_{x, y, y^{\prime}}(A)$ and $u:=x$ implies that $\Psi_{x}\left(\varphi_{x, y, y^{\prime}}(A)\right) \neq \emptyset$. Clearly, the set $\Psi_{x}\left(\varphi_{x, y, y^{\prime}}(A)\right)$ is closed and convex. Therefore, the set-valued mapping $\mathcal{B} \ni$ $A \mapsto \Psi_{x}\left(\varphi_{x, y, y^{\prime}}(A)\right) \in \mathcal{B}$ has nonempty closed convex values and also a closed graph (this last property holds because $\Psi_{x}$ has a closed graph and $\varphi_{x, y, y^{\prime}}$ is continuous). Since $\mathcal{B}$ is compact and convex, we can apply Gliksberg's extension of the Kakutani fixed point theorem given in [16] to obtain the claimed property.

Final part of the proof of Theorem 7. In the last part of the proof we will show that the mapping $(f+F)^{-1}$ has the Aubin property at 0 for $\bar{x}$; then, according to the equivalence of this last property with metric regularity of $f+F$ at $\bar{x}$ for 0 , we will arrive at the desired result. Specifically, we will show that for any $y, y^{\prime} \in \mathbb{B}_{\varepsilon b}(0)$ and any $x^{\prime} \in(f+F)^{-1}\left(y^{\prime}\right) \cap \mathbb{B}_{\delta}(\bar{x})$, there exists $x \in(f+F)^{-1}(y)$ such that

$$
\left\|x-x^{\prime}\right\| \leq \frac{\ell}{1-\varepsilon \ell}\left\|y-y^{\prime}\right\| .
$$

Taking into account the choice of the constants $\ell$ and $\varepsilon$, this will give us (17).

To show (34), we construct a sequence $\left\{x_{n}\right\}$ in $X$ and a sequence $\left\{A_{n}\right\}$ in $\mathcal{B}$ that satisfy for each nonnegative integer $n$ the following relations:

(i) $\left\|x_{n}-\bar{x}\right\|<3 \delta$;

(ii) $\left\|x_{n+1}-x_{n}\right\| \leq(\varepsilon \ell)^{n}\left\|x_{1}-x_{0}\right\|$;

(iii) $\left\|f\left(x_{n+1}\right)-f\left(x_{n}\right)-A_{n}\left(x_{n+1}-x_{n}\right)\right\| \leq \varepsilon\left\|x_{n+1}-x_{n}\right\|$;

(iv) $f\left(x_{n}\right)+A_{n}\left(x_{n+1}-x_{n}\right)+F\left(x_{n+1}\right) \ni y$.

We use induction. Let $x_{0}:=x^{\prime}$. Since $x_{0} \in(f+F)^{-1}\left(y^{\prime}\right) \cap \mathbb{B}_{\delta}(\bar{x})$, by Lemma 14 the mapping $\Psi_{x_{0}} \circ \varphi_{x_{0}, y, y^{\prime}}$ has a fixed point $A_{0} \in \mathcal{B}$. Set $x_{1}:=x_{0}+\varphi_{x_{0}, y, y^{\prime}}\left(A_{0}\right)$. Then $A_{0}=\Psi_{x_{0}}\left(x_{1}-x_{0}\right)$, hence

$$
\left\|f\left(x_{1}\right)-f\left(x_{0}\right)-A_{0}\left(x_{1}-x_{0}\right)\right\| \leq \varepsilon\left\|x_{1}-x_{0}\right\|,
$$

which is (iii) with $n=0$. Note that (i) and (ii) with $n=0$ hold trivially. Further, from

$$
x_{1}=x_{0}+\varphi_{x_{0}, y, y^{\prime}}\left(A_{0}\right) \in G_{A_{0}}^{-1}\left(y-f\left(x_{0}\right)+f(\bar{x})+A_{0}\left(x_{0}-\bar{x}\right)\right) \cap \mathbb{B}_{\ell\left\|y-y^{\prime}\right\|}\left(x_{0}\right),
$$

we obtain (iv) for $n=0$. Moreover, we have

$$
\left\|x_{1}-x_{0}\right\| \leq \ell\left\|y-y^{\prime}\right\| \leq 2 \ell \varepsilon b<2 b<2 \delta .
$$

Hence $\left\|x_{1}-\bar{x}\right\| \leq\left\|x_{1}-x_{0}\right\|+\left\|x_{0}-\bar{x}\right\|<3 \delta$, which is (i) with $n=1$.

Further, suppose that for a positive integer $N$ we have found $x_{0}, x_{1}, \ldots, x_{N}$ and $A_{0}, \ldots, A_{N-1}$ that satisfy conditions (i)-(iv) for all $n<N$ and (i) with $n=N$.

By (i) with $n=N$ we have $x_{N} \in \mathbb{B}_{3 \delta}(\bar{x})$. By (iv) for $n=N-1$, we obtain

$$
y_{N}^{\prime}:=y+f\left(x_{N}\right)-f\left(x_{N-1}\right)-A_{N-1}\left(x_{N}-x_{N-1}\right) \in f\left(x_{N}\right)+F\left(x_{N}\right) .
$$


Thus $x_{N} \in(f+F)^{-1}\left(y_{N}^{\prime}\right) \cap \mathbb{B}_{3 \delta}(\bar{x})$. Combining (ii) and (iii) for $n=N-1$ with (35) we get

$$
\begin{aligned}
\left\|y_{N}^{\prime}\right\| & \leq\|y\|+\left\|f\left(x_{N}\right)-f\left(x_{N-1}\right)-A_{N-1}\left(x_{N}-x_{N-1}\right)\right\| \leq \varepsilon b+\varepsilon\left\|x_{N}-x_{N-1}\right\| \\
& \leq \varepsilon b+\varepsilon\left\|x_{1}-x_{0}\right\|<\varepsilon b+2 \varepsilon b=3 \varepsilon b .
\end{aligned}
$$

From Lemma 14 we conclude that the mapping $\Psi_{x_{N}} \circ \varphi_{x_{N}, y, y_{N}^{\prime}}$ has a fixed point in $\mathcal{B}$; denote it by $A_{N}$. Set $x_{N+1}:=x_{N}+\varphi_{x_{N}, y, y_{N}^{\prime}}\left(A_{N}\right)$. Then $A_{N}=\Psi_{x_{N}}\left(x_{N+1}-x_{N}\right)$, hence

$$
\left\|f\left(x_{N+1}\right)-f\left(x_{N}\right)-A_{N}\left(x_{N+1}-x_{N}\right)\right\| \leq \varepsilon\left\|x_{N+1}-x_{N}\right\|,
$$

which is (iii) for $n=N$. Note that

$$
\begin{aligned}
x_{N+1} & =x_{N}+\varphi_{x_{N}, y, y_{N}^{\prime}}\left(A_{N}\right) \\
& =G_{A_{N}}^{-1}\left(y-f\left(x_{N}\right)+f(\bar{x})+A_{N}\left(x_{N}-\bar{x}\right)\right) \cap \mathbb{B}_{\ell\left\|y-y_{N}^{\prime}\right\|}\left(x_{N}\right),
\end{aligned}
$$

hence (iv) is satisfied for $n=N$. Noting that (iii) and (ii) with $n=N-1$ imply

$$
\begin{aligned}
\left\|x_{N+1}-x_{N}\right\| & \leq \ell\left\|y-y_{N}^{\prime}\right\|=\ell\left\|f\left(x_{N}\right)-f\left(x_{N-1}\right)-A_{N-1}\left(x_{N}-x_{N-1}\right)\right\| \\
& \leq \varepsilon \ell\left\|x_{N}-x_{N-1}\right\| \leq \varepsilon \ell(\varepsilon \ell)^{N-1}\left\|x_{1}-x_{0}\right\|,
\end{aligned}
$$

we obtain that (ii) holds for $n=N$. By (35) and (28), we also have

$$
\begin{aligned}
\left\|x_{N+1}-\bar{x}\right\| & \leq\left\|x_{0}-\bar{x}\right\|+\sum_{n=0}^{N}\left\|x_{n+1}-x_{n}\right\|<\delta+\frac{\left\|x_{1}-x_{0}\right\|}{1-\ell \varepsilon} \leq \delta+\frac{2 \ell \varepsilon b}{1-\ell \varepsilon} \\
& =\delta+2 \ell \varepsilon \delta<3 \delta .
\end{aligned}
$$

We arrive at (i) for $n=N+1$. The induction step is complete.

Since $x^{\prime}=x_{0}$, the combination of (ii) and (35) implies that, for each natural $n$,

$$
\left\|x_{n}-x^{\prime}\right\| \leq \sum_{i=0}^{n-1}\left\|x_{i+1}-x_{i}\right\| \leq \frac{\left\|x_{1}-x^{\prime}\right\|}{1-\ell \varepsilon} \leq \frac{\ell}{1-\ell \varepsilon}\left\|y^{\prime}-y\right\| .
$$

Since $\left\{x_{n}\right\}$ is a Cauchy sequence, it converges to some $x \in X$. From (iv), (i), and (29) we get for each index $n$ that

$$
\begin{aligned}
\left(x_{n+1}, y-f\left(x_{n}\right)+A_{n}\left(x_{n}-x_{n+1}\right)\right) & \in\left(\mathbb{B}_{3 \delta}(\bar{x}) \times \mathbb{B}_{\beta}(-f(\bar{x}))\right) \cap \operatorname{gph} F \\
& \subset\left(\mathbb{B}_{2 \ell \beta}(\bar{x}) \times \mathbb{B}_{\beta}(-f(\bar{x}))\right) \cap \operatorname{gph} F .
\end{aligned}
$$

Since the last set is closed, the continuity of $f$ and the boundedness of the set $\mathcal{B}$ where $A_{n}$ belong, imply that, passing to the limit, we have $(x, y-f(x)) \in \operatorname{gph} F$, that is, $y \in f(x)+F(x)$. Taking the limit with $n$ in (36) we complete the proof of (34).

4. Two corollaries. In this section we will show that the main results of the recent papers [4] and [6] can be derived from Theorem 7 . The following theorem is a slightly improved version of the main result in [4] also including an estimate for the regularity modulus.

THEOREM 15. Consider a function $f: X \rightarrow Y$, a set-valued mapping $F: X \rightarrow Y$, and a point $(\bar{x}, \bar{y}) \in \operatorname{gph}(f+F)$ with $\bar{x} \in \operatorname{int} \operatorname{dom} f$ and suppose that for a convex 
subset $\mathcal{T}$ of $\mathcal{L}(X, Y)$ and a constant $\mu \geq 0$ the assumptions (A) in Theorem 6 and (B) in Theorem 7 are satisfied. In addition, suppose that assumption (B) is augmented by the condition that for every $A \in \mathcal{T}$ the mapping $G_{A}$ in (12) is strongly metrically regular at $\bar{x}$ for $\bar{y}$. Then the mapping $f+F$ is strongly metrically regular at $\bar{x}$ for $\bar{y}$; moreover, its regularity modulus satisfies (17).

Proof. On the assumptions of Theorem 15, there are positive constants $\varepsilon$ and $\ell$ such that $(23)$ holds. Find $\mathcal{A}=\left\{A_{1}, A_{2}, \ldots, A_{k}\right\} \subset \mathcal{T}$ such that $\mathcal{T} \subset \mathcal{A}+(\varepsilon-\mu) \mathbb{B}$. For each $i \in\{1,2, \ldots, k\}$, the strong metric regularity of $G_{A_{i}}$ yields the existence of $\beta_{i}>0$ such that the mapping $\mathbb{B}_{\beta_{i}}(\bar{y}) \ni w \mapsto G_{A_{i}}^{-1}(w) \cap \mathbb{B}_{\ell \beta_{i}}(\bar{x})$ is single-valued and Lipschitz continuous with the constant $\ell$. Let $\beta:=\min \beta_{i}$.

We will now show that for some $b>0$ the set $G_{A}^{-1}(v) \cap \mathbb{B}_{b}(\bar{x})$ is at most singleton for each $v \in \mathbb{B}_{b}(\bar{y})$ and each $A \in \mathcal{T}$. Since $\mathcal{T}$ is bounded, there is $b \in(0, \beta \min \{1, \ell\})$ such that

$$
v+\left(A^{\prime}-A^{\prime \prime}\right)(u-\bar{x}) \in \mathbb{B}_{\beta}(\bar{y}) \quad \text { whenever } \quad\left(u, v, A^{\prime}, A^{\prime \prime}\right) \in \mathbb{B}_{b}(\bar{x}) \times \mathbb{B}_{b}(\bar{y}) \times \mathcal{A} \times \mathcal{T} .
$$

Fix arbitrary $v \in \mathbb{B}_{b}(\bar{y})$ and $A \in \mathcal{T}$. Suppose that there are two distinct $u, u^{\prime} \in$ $G_{A}^{-1}(v) \cap \mathbb{B}_{b}(\bar{x})$. Pick $A_{i} \in \mathcal{A}$ with $\left\|A_{i}-A\right\| \leq \varepsilon-\mu$. Then both $w:=v+\left(A_{i}-A\right)(u-\bar{x})$ and $w^{\prime}:=v+\left(A_{i}-A\right)\left(u^{\prime}-\bar{x}\right)$ are in $\mathbb{B}_{\beta}(\bar{y}) \subset \mathbb{B}_{\beta_{i}}(\bar{y})$ and also $u \in G_{A_{i}}^{-1}(w) \cap \mathbb{B}_{\ell \beta_{i}}(\bar{x})$ and $u^{\prime} \in G_{A_{i}}^{-1}\left(w^{\prime}\right) \cap \mathbb{B}_{\ell \beta_{i}}(\bar{x})$. Thus

$$
0<\left\|u-u^{\prime}\right\| \leq \ell\left\|w-w^{\prime}\right\|=\ell\left\|\left(A_{i}-A\right)\left(u-u^{\prime}\right)\right\| \leq \ell(\varepsilon-\mu)\left\|u-u^{\prime}\right\|<\left\|u-u^{\prime}\right\|,
$$

which is impossible. Hence $G_{A}^{-1}(v) \cap \mathbb{B}_{b}(\bar{x})$ is at most singleton. Thus, all assumptions of Theorem 7 hold, hence the mapping $f+F$ is metrically regular at $\bar{x}$ for $\bar{y}$ with regularity modulus satisfying (17).

Since

$$
\kappa:=\ell /(1-\varepsilon \ell)>B /(1-(\mu+\chi(\mathcal{T})) B),
$$

for any sufficiently small $\gamma>0$ the mapping

$$
\mathbb{B}_{\gamma}(\bar{y}) \ni y \mapsto \sigma_{\gamma}(y):=(f+F)^{-1}(y) \cap \mathbb{B}_{\kappa \gamma}(\bar{x})
$$

is a non-empty-valued localization of $(f+F)^{-1}$ around $\bar{y}$ for $\bar{x}$. But $f$ is continuous and $\mathcal{T}$ is bounded, hence there is $\gamma \in\left(0, \kappa^{-1} \min \{r, \ell \beta\}\right)$, where $r$ is the constant from (A), such that

$$
y-f(x)+f(\bar{x})+A(x-\bar{x}) \in \mathbb{B}_{\beta}(\bar{y}) \quad \text { for each } \quad(x, y, A) \in \mathbb{B}_{\kappa \gamma}(\bar{x}) \times \mathbb{B}_{\gamma}(\bar{y}) \times \mathcal{T} .
$$

It suffices to show that $\sigma_{\gamma}$ is nowhere multivalued on $\mathbb{B}_{\gamma}(\bar{y})$; then, from [12, Proposition 3G.1], $f+F$ is in fact strongly metrically regular at $\bar{x}$ for $\bar{y}$.

Suppose that there exists $y \in \mathbb{B}_{\gamma}(\bar{y})$ for which there are two distinct $x^{\prime}, x^{\prime \prime} \in \sigma_{\gamma}(y)$. Since $\kappa \gamma<r$, assumption (A) yields the existence of $A \in \mathcal{T}$ such that

$$
\left\|f\left(x^{\prime}\right)-f\left(x^{\prime \prime}\right)-A\left(x^{\prime}-x^{\prime \prime}\right)\right\| \leq \mu\left\|x^{\prime}-x^{\prime \prime}\right\| .
$$

Let $A_{i} \in \mathcal{A}$ be such that $\left\|A_{i}-A\right\| \leq \varepsilon-\mu$. Then

$$
\left\|f\left(x^{\prime}\right)-f\left(x^{\prime \prime}\right)-A_{i}\left(x^{\prime}-x^{\prime \prime}\right)\right\| \leq \varepsilon\left\|x^{\prime}-x^{\prime \prime}\right\| .
$$


From (37), both $w^{\prime}:=y-f\left(x^{\prime}\right)+f(\bar{x})+A_{i}\left(x^{\prime}-\bar{x}\right)$ and $w^{\prime \prime}:=y-f\left(x^{\prime \prime}\right)+f(\bar{x})+$ $A_{i}\left(x^{\prime \prime}-\bar{x}\right)$ are in $\mathbb{B}_{\beta}(\bar{y})$. Since $\kappa \gamma<\ell \beta \leq \ell \beta_{i}$ and $x^{\prime}, x^{\prime \prime} \in(f+F)^{-1}(y) \cap \mathbb{B}_{\kappa \gamma}(\bar{x})$ we obtain that

$$
x^{\prime}=G_{A_{i}}^{-1}\left(w^{\prime}\right) \cap \mathbb{B}_{\ell \beta_{i}}(\bar{x}) \quad \text { and } \quad x^{\prime \prime}=G_{A_{i}}^{-1}\left(w^{\prime \prime}\right) \cap \mathbb{B}_{\ell \beta_{i}}(\bar{x}) .
$$

Taking the difference gives us

$$
\begin{aligned}
& 0<\left\|x^{\prime}-x^{\prime \prime}\right\| \leq \ell\left\|w^{\prime}-w^{\prime \prime}\right\|=\ell\left\|f\left(x^{\prime}\right)-f\left(x^{\prime \prime}\right)-A_{i}\left(x^{\prime}-x^{\prime \prime}\right)\right\| \\
& \leq \ell \varepsilon\left\|x^{\prime}-x^{\prime \prime}\right\|<\left\|x^{\prime}-x^{\prime \prime}\right\|,
\end{aligned}
$$

which is a contradiction. Hence, $\sigma_{\gamma}$ is not multivalued on its domain and the proof is complete.

There are some parts of the proof of Theorem 15 in [4] that are similar to parts of the proof of Theorem 7 in the present paper but there are also important differences. For example, in [4] we used Brouwer's fixed point theorem instead of Gliksberg's extension of the Kakutani fixed point theorem, which allows us to shorten the argument in Lemma 14 in comparison to the one used in [4, Lemma 3]. We also use a different iteration procedure relying on the new Lemma 13.

We will next show how to derive the main result in [6] from Theorem 7 . In the proof of Theorem 7 it is not really needed to assume that $f$ is defined on the whole neighborhood of $\bar{x}$. It suffices to assume that $\operatorname{dom} f \supset \operatorname{dom} F \cap \mathbb{B}_{r}(\bar{x})=: D$ for some $r>0$ and suppose that (A) holds only for $u, v \in D$.

Theorem 16. Let $X$ and $Y$ be Banach spaces, and let $f: X \rightarrow Y$ be a continuous mapping with closed convex domain. Assume that for a given $\bar{x} \in \operatorname{dom} f$ there is a compact convex subset $\mathcal{T}$ of $\mathcal{L}(X, Y)$ along with positive $\varrho$ and $\mu$ such that

(a) there exists a neighborhood $U$ of $\bar{x}$ such that for any $x, x^{\prime} \in U \cap \operatorname{dom} f$ there is $A \in \mathcal{T}$ satisfying

$$
\left\|f(x)-f\left(x^{\prime}\right)-A\left(x-x^{\prime}\right)\right\| \leq \mu\left\|x-x^{\prime}\right\| ;
$$

(b) $(\varrho+\mu) \mathbb{B} \subset A(\mathbb{B} \cap(\operatorname{dom} f-\bar{x}))$ for any $A \in \mathcal{T}$.

Then $f$ is metrically regular at $\bar{x}$ with $\operatorname{reg}(f ; \bar{x}) \leq 1 / \varrho$.

Proof. Without any loss of generality assume that $\bar{x}=0$ and $f(\bar{x})=0$. Let $r>0$ be such that (a) holds for any $x, x^{\prime} \in(r \mathbb{B}) \cap \operatorname{dom} f=: D$. Define $F: X \rightrightarrows Y$ by $F(x)=0$ when $x \in \operatorname{dom} f$, and $F=\emptyset$ otherwise. Then $f=f+F$ and (A) holds for $u, v \in D$. Fix any $A \in \mathcal{T}$. The mapping $G_{A}$ from (12) is just the restriction of $A$ to $\operatorname{dom} f$. Thus, it satisfies the convexity assumption in (C) for $U \times V:=X \times Y$. By (b), $\operatorname{reg}\left(G_{A} ; 0\right) \leq 1 /(\varrho+\mu)$. Indeed, let $\varrho^{\prime} \in(0, \varrho)$ be arbitrary. Pick $\gamma \in(0,1)$ such that $\mu+\varrho^{\prime}<(1-\gamma)(\mu+\varrho)$. There is a constant $\delta \in(0,1)$ such that for each $x \in \delta \mathbb{B}$ we have

$$
(1-\gamma) \mathbb{B}-x \subset \mathbb{B} \quad \text { and } \quad\|A x\|<(1-\gamma)(\mu+\varrho)-\mu-\varrho^{\prime} .
$$

Fix any $x \in(\delta \mathbb{B}) \cap \operatorname{dom} f$. The convexity of $\operatorname{dom} f$ implies that

$$
\begin{aligned}
A(\mathbb{B} \cap(\operatorname{dom} f-x)) & \supset A(((1-\gamma) \mathbb{B}) \cap \operatorname{dom} f-x) \supset A((1-\gamma)[\mathbb{B} \cap \operatorname{dom} f])-A x \\
& \supset(1-\gamma)(\mu+\varrho) \mathbb{B}-A x \supset\left(\mu+\varrho^{\prime}\right) \mathbb{B} .
\end{aligned}
$$

Fix $\varepsilon \in(0, \delta)$. Since $\varepsilon<1$, the convexity of $\operatorname{dom} f-x$ implies that

$$
\begin{aligned}
A((x+\varepsilon \mathbb{B}) \cap \operatorname{dom} f) & =A x+A((\varepsilon \mathbb{B}) \cap(\operatorname{dom} f-x)) \\
& \supset A x+A(\varepsilon[\mathbb{B} \cap(\operatorname{dom} f-x)]) \supset A x+\left(\mu+\varrho^{\prime}\right) \varepsilon \mathbb{B} .
\end{aligned}
$$


Thus, (4) holds with $\lambda:=\mu+\varrho^{\prime}$. Since $\varrho^{\prime}<\varrho$ was chosen arbitrarily we obtain the desired estimate for $\operatorname{reg}\left(G_{A} ; 0\right)$.

Noting that $\mathcal{T}$ is compact, we have $\chi(\mathcal{T})=0$ and thus (B) is satisfied. Applying Theorem 7 we conclude that $f$ is metrically regular at 0 , and

$$
\operatorname{reg}(f ; 0) \leq\left(\left((\varrho+\mu)^{-1}\right)^{-1}-\mu\right)^{-1}=\varrho^{-1} .
$$

5. Applications. In this section we present applications of Theorem 7. First, we consider a special case where the function $f$ in Theorem 7 is defined by a Nemytskii operator. Let $L_{\infty}^{k}(0,1)$ be the space of all measurable and essentially bounded functions $x(\cdot)$ defined on $[0,1]$ with values in $\mathbb{R}^{k}$, for some natural $k$, and the standard norm

$$
\|x(\cdot)\|_{\infty}=\operatorname{ess}_{\sup } \operatorname{se}_{t 0,1]}\|x(t)\|
$$

and let $X$ be a Banach space which is a subspace of $L_{\infty}^{k}(0,1)$ and is equipped with a norm $\|\cdot\|$ stronger than $\|\cdot\|_{\infty}$; that is, for any $x \in X$ one has $\|x\| \geq\|x\|_{\infty}$. Setting $Y=L_{\infty}^{s}(0,1)$, the mapping $f: X \rightarrow Y$ defined as

$$
f(x)(t)=\varphi(x(t)),
$$

where $\varphi: \mathbb{R}^{k} \rightarrow \mathbb{R}^{s}$ is locally Lipschitz continuous, is usually called a Nemytskii operator; see, e.g., [27]. Recall that the Clarke's generalized Jacobian $\bar{\partial} \varphi(\xi)$ of $\varphi$ at $\xi \in \mathbb{R}^{k}$ consists of $(s \times k)$-matrices.

Let $\bar{x} \in X, \delta>0$, and $\varepsilon \geq 0$, and let $\mathcal{D}=\mathcal{D}_{\delta, \varepsilon}^{\bar{x}}$ be a measurable, closed- and convex-valued mapping, $\mathcal{D}:[0,1] \rightrightarrows \mathbb{R}^{n}$, having the following property.

Property (P). For a.e. $t \in[0,1]$, for every $\xi \in \mathbb{B}_{\delta}(\bar{x}(t))$, and for every $D^{\prime} \in \bar{\partial} \varphi(\xi)$, there exists $D \in \mathcal{D}(t)$ such that $\left\|D-D^{\prime}\right\| \leq \varepsilon$ (where we use the operator norm).

Let $\mathcal{T}=\mathcal{T}_{\delta, \varepsilon}^{\bar{x}}$ be the set of all measurable selections of $\mathcal{D}$. Notice that every $A \in \mathcal{T}$ is a measurable and bounded $(s \times k)$-matrix function of $t$; thus it can be viewed as an element of $\mathcal{L}(X, Y)$, acting as $(A x)(t)=A(t) x(t), x \in X$. Consider a set-valued mapping $F: X \rightrightarrows Y$ with closed and convex graph and a point $\bar{y} \in(f+F)(\bar{x})$.

Proposition 17. Let $\varphi, \mathcal{D}$, and $\mathcal{T}$ be as described. Assume that for every $A \in \mathcal{T}$ the mapping $G_{A}$ in (12) is metrically regular at $\bar{x}$ for $\bar{y}$ and, in addition, $\beta(\varepsilon+\chi(\mathcal{T}))<$ 1 , where $\mathrm{B}$ is defined in (13). Then

$$
\operatorname{reg}(f+F ; \bar{x} \mid \bar{y}) \leq\left(B^{-1}-(\varepsilon+\chi(\mathcal{T}))^{-1} .\right.
$$

Proof. We have to check conditions $(\mathrm{A})-(\mathrm{C})$ stated in Theorems 6 and 7 with $\mu=\varepsilon$ and $r=\delta$. Condition (C) holds since $F$ has convex graph. Condition (B) is an assumption. To check condition (A) we take arbitrary $u, v \in \mathbb{B}_{\delta}(\bar{x})$ and consider the difference $f(u)(t)-f(v)(t)=\varphi(u(t))-\varphi(v(t))$. Fix $t \in[0,1]$ for which $u(t), v(t) \in$ $\mathbb{B}_{\delta}(\bar{x}(t))$. According to the mean value theorem [8, Proposition 2.6.5] there exists $D_{t}^{\prime} \in \operatorname{co} \bar{\partial} \varphi(\operatorname{co}\{u(t), v(t)\})=: \Xi(t)$ such that

$$
\varphi(u(t))-\varphi(v(t))=D_{t}^{\prime}(u(t)-v(t)) .
$$

One may use the representation $D_{t}^{\prime}=\sum_{i=1}^{n} \alpha_{i} D_{t i}^{\prime}$, where $n \leq s k+1, \alpha_{i} \geq 0$, $\sum_{i=1}^{n} \alpha_{i}=1, D_{t i}^{\prime} \in \bar{\partial} \varphi\left(\xi_{i}\right), \xi_{i} \in \operatorname{co}\{u(t), v(t)\} \subset \mathbb{B}_{\delta}(\bar{x}(t))$. Property (P) implies that 
there exist $D_{t i} \in \mathcal{D}(t)$ such that $\left\|D_{t i}-D_{t i}^{\prime}\right\| \leq \varepsilon, i=1, \ldots, n$. Then $D_{t}:=\sum_{i=1}^{n} \alpha_{i} D_{t i}$ satisfies $D_{t} \in \mathcal{D}(t)$ and $\left\|D_{t}-D_{t}^{\prime}\right\| \leq \varepsilon$. Define

$$
\begin{gathered}
\Gamma(t)=\left\{\left(D, D^{\prime}\right) \mid D^{\prime} \in \Xi(t), \varphi(u(t))-\varphi(v(t))=D^{\prime}(u(t)-v(t)), D \in \mathcal{D}(t),\right. \\
\left.\left\|D-D^{\prime}\right\| \leq \varepsilon\right\} .
\end{gathered}
$$

The set $\Gamma(t)$ is nonempty since it contains $\left(D_{t}, D_{t}^{\prime}\right)$. This applies for a.e. $t \in[0,1]$. The outer semicontinuity of $\bar{\partial} \varphi$ [8, Proposition 2.6.2] implies that $\Xi(t)$ is closed, which together with the closedness of $\mathcal{D}(t)$ gives closedness of $\Gamma(t)$. Moreover, the mapping $t \mapsto \Gamma(t)$ is measurable. Indeed, the mapping $t \mapsto \Xi(t)$ is measurable due to the outer semicontinuity of $\bar{\partial} \varphi$ and the fact that taking a convex hull preserves measurability. Then the measurability of $\Gamma$ follows from [1, Theorem 8.2.9]. Hence, $\Gamma$ has a measurable selection $\left(A(t), A^{\prime}(t)\right)$. In particular, $A \in \mathcal{T}$ by the definition of $\mathcal{T}$. Then

$$
\begin{aligned}
\|f(u)-f(v)-A(u-v)\|_{\infty}= & \operatorname{esssup}_{t \in[0,1]}\|\varphi(u(t))-\varphi(v(t))-A(t)(u(t)-v(t))\| \\
\leq & \operatorname{ess\operatorname {sup}}\left(\left\|\varphi(u(t))-\varphi(v(t))-A^{\prime}(t)(u(t)-v(t))\right\|\right. \\
& +\varepsilon\|u(t)-v(t)\|) \\
= & \varepsilon\|u-v\|_{\infty} \leq \varepsilon\|u-v\| .
\end{aligned}
$$

Thus, condition (A) holds as well. Theorem 7 then implies the estimate (39).

Note that the measure of noncompactness $\chi(\mathcal{T})$ can be estimated as follows:

$$
\chi(\mathcal{T}) \leq \chi:=\sup _{t \in[0,1]} \min _{D \in \mathcal{D}(t)} \max _{D^{\prime} \in \mathcal{D}(t)}\left\|D-D^{\prime}\right\|=\sup _{t \in[0,1]} \operatorname{rad} D(t) .
$$

This is an easy consequence of [1, Theorem 8.2.11], which implies existence of a measurable selection $D(t) \in \mathcal{D}(t)$ (thus $D(\cdot) \in \mathcal{T}$ ) with $\|D(t)-A(t)\| \leq \chi$ for every $A \in \mathcal{T}$.

Corollary 18. Assume that $\bar{\partial} \varphi$ is uniformly outer semicontinuous around the set $\bar{x}([0,1])$, meaning that for every $\varepsilon>0$ there exists $\delta>0$ such that for a.e. $t \in[0,1]$ it holds that $\bar{\partial} \varphi(\xi) \subset \bar{\partial} \varphi(\bar{x}(t))+\varepsilon \mathbb{B}$ whenever $\|\xi-\bar{x}(t)\| \leq \delta$. Let $\mathcal{T}$ be the set of all measurable selections of the mapping $t \mapsto \bar{\partial} \varphi(\bar{x}(t))$. Assume also that for every $A \in \mathcal{T}$ the mapping $G_{A}$ defined in (12) is metrically regular at $\bar{x}$ for $\bar{y}$ and $B \chi(\mathcal{T})<1$, where $B$ is defined in (13). Then

$$
\operatorname{reg}(f+F ; \bar{x} \mid \bar{y}) \leq\left(\beta^{-1}-\chi(\mathcal{T})\right)^{-1} .
$$

Proof. It is enough to observe that for every $\varepsilon>0$ there is $\delta>0$ such that Property $(\mathrm{P})$ is fulfilled for the mapping $\mathcal{T}$ (which is independent of $\varepsilon$ ). Then Proposition 17 yields metric regularity of $f+F$, and the estimation for $\operatorname{reg}(f+F ; \bar{x} \mid \bar{y})$ follows from (39), since the latter holds for any $\varepsilon>0$.

If, in particular, $\varphi$ is continuously differentiable, we have $\chi(\mathcal{T})=0$ in (41) since $\mathcal{T}=\{\nabla \varphi(\bar{x}(\cdot))\}$, and then $\operatorname{reg}(f+F ; \bar{x} \mid \bar{y}) \leq \beta$.

If the generalized Jacobian $\bar{\partial} \varphi$ is not uniformly outer semicontinuous around $\bar{x}([0,1])$ (or this property is not easy to check) it is still possible to define the mapping $\mathcal{D}$ in such a way that Property $(\mathrm{P})$ holds with an arbitrarily small $\delta>0$ and $\varepsilon=0$; namely, we put

$$
\mathcal{D}_{\delta}(t)=\overline{\mathrm{co}} \bigcup_{\xi \in \mathbb{B}_{\delta}(\bar{x}(t))} \bar{\partial} \varphi(\xi)
$$


The measurability of this mapping follows from the outer semicontinuity of $\bar{\partial} \varphi$. Observe that $\mathcal{D}_{\delta}$ has Property $(\mathrm{P})$ with $\varepsilon=0$. Applying Proposition 17 we obtain the following corollary, where as before we define $\mathcal{T}_{\delta} \subset \mathcal{L}(X, Y)$ as the set of all measurable selections of $\mathcal{D}_{\delta}$.

Corollary 19. Let the mapping $G_{A}$ defined in (12) be metrically regular at $\bar{x}$ for $\bar{y}$ for every $A \in \mathcal{T}_{\delta}$, and let $\beta \chi\left(\mathcal{T}_{\delta}\right)<1$, where $\beta:=\sup _{A \in \mathcal{T}_{\delta}} \operatorname{reg}\left(G_{A} ; \bar{x} \mid \bar{y}\right)$. Then

$$
\operatorname{reg}(f+F ; \bar{x} \mid \bar{y}) \leq\left(\Re^{-1}-\chi\left(\mathcal{T}_{\delta}\right)\right)^{-1} .
$$

Note that the mapping $\mathcal{T}_{\delta}$ can be larger than $\mathcal{T}$ in Corollary 18.

We now apply the results just obtained for the Nemytskii operator to establish conditions for metric regularity of a feasibility mapping in control. Consider a controlled ODE of the form

$$
\dot{p}(t)=g(p(t), u(t)), \quad t \in I:=[0,1] .
$$

The control function $u: I \rightarrow \mathbb{R}^{d}$ is an element of the space $L_{\infty}^{d}$ of measurable and essentially bounded functions, the state function $p: I \rightarrow \mathbb{R}^{n}$ is an element of $W_{1, \infty}^{n, 0}$, the space of Lipschitz continuous functions with $p(0)=0$. A pair $x=(p, u) \in X:=$ $W_{1, \infty}^{n, 0} \times L_{\infty}^{d}$ which satisfies (44) almost everywhere on $I$ together with the pointwise constraint

$$
C(p(t), u(t)) \leq 0 \text { for a.e. } t \in I
$$

is said to be a feasible process. The functions $g: \mathbb{R}^{n+d} \rightarrow \mathbb{R}^{n}$ and $C: \mathbb{R}^{n+d} \rightarrow \mathbb{R}^{l}$ are assumed to be locally Lipschitz continuous everywhere. In (45) and further the notation $h \leq 0$ for a vector $h=\left(h_{1}, h_{2}, \ldots, h_{l}\right) \in \mathbb{R}^{l}$ means that $h_{i} \leq 0$ for each $i \in\{1,2, \ldots, l\}$.

System (44)-(45) can be written in the form of the generalized equation

$$
0 \in f(x)+F(x), \text { with } x=(p, u), f(x)=\left(\begin{array}{c}
g(p, u) \\
C(p, u)
\end{array}\right) \text { and } F(x)=\left(\begin{array}{c}
-\dot{p} \\
\mathbb{R}_{+}^{l}
\end{array}\right) .
$$

More precisely, $F(x)$ is defined as

$$
\left\{(\xi, \nu) \in L_{\infty}^{n} \times L_{\infty}^{l} \mid \xi(t)=-\dot{p}(t), \nu(t) \geq 0 \text { for a.e. } t \in[0,1]\right\} .
$$

The set $(f+F)^{-1}(0)$ consists of all feasible processes; therefore the mapping $f+F$ is said to be the feasibility mapping.

Establishing metric regularity of the mapping $f+F$ is of fundamental importance in control. First of all, metric regularity is a basic tool in deriving necessary conditions of optimality, which in optimal control are usually called Pontryagin's maximum principle. Furthermore, metric regularity provides a basis for estimating the sensitivity of the feasibility mapping and allows one to apply various numerical techniques.

Observe that $f$ is in Nemytskii form (38) with $\varphi=(g, C): \mathbb{R}^{n+d} \rightarrow \mathbb{R}^{n+l}$. Let $\bar{x}=(\bar{p}, \bar{u})$ be a feasible process. For $\delta>0$ define $\mathcal{D}_{\delta}(t)$ as in (42). In the case when both $g$ and $C$ are continuously differentiable, we can replace $\mathcal{D}_{\delta}(t)$ by $\mathcal{D}(t)=\{(\nabla g(\bar{x}(t)), \nabla C(\bar{x}(t)))\}$ and then eliminate $\delta$ in all further considerations. Let $\mathcal{T}_{\delta} \subset \mathcal{L}(X, Y)$ be the set of all measurable selections of $\mathcal{D}_{\delta}$. Then any $A \in \mathcal{T}_{\delta}$ has the structure

$$
A(t)=\left(\begin{array}{cc}
P(t) & Q(t) \\
R(t) & S(t)
\end{array}\right)
$$


where $P(t)$ has dimension $(n \times n), Q(t)$ has dimension $(n \times d)$, etc., and these submatrices depend on the choice of $A \in \mathcal{T}_{\delta}$.

To state the result given next we need some notation. First, in finite-dimensional spaces the Euclidean norm is used for vectors and the corresponding operator norm is used for matrices. The norm in $X$ is the sum of the $W_{1, \infty}$ and the $L_{\infty}$ norms, and similarly for $Y$. Consider the equation $\dot{p}=P p+\xi, p(0)=0$, with $A \in \mathcal{T}_{\delta}$ and $\xi \in L_{\infty}^{n}$. Its solution has the form $p=L \xi$, where $L$ is a linear continuous operator from $L_{\infty}$ to $W_{1, \infty}^{n, 0}$. Clearly, both $L$ and $R$ depend on the choice of $A$; furthermore, from the local Lipschitz continuity of $(g, C)$ the quantities

$$
\Delta:=\sup _{A \in \mathcal{T}_{\delta}}\|L\|_{\infty} \quad \text { and } \quad \rho:=\sup _{A \in \mathcal{T}_{\delta}}\|R\|_{\infty}
$$

are finite; see (47) for the relation between $A$ and $P, R$. The following theorem gives a sufficient condition for metric regularity of the feasibility mapping.

Theorem 20. Assume that for some $\delta>0, \alpha>0$, and $\gamma \in(0,1)$ the set $\mathcal{T}_{\delta}$ has the following property: for every $A \in \mathcal{T}_{\delta}$ (see (47) for the structure of $A$ ) there exist functions $w \in W_{1, \infty}^{n}$ and $v \in L_{\infty}^{d}$ with $\|(w, v)\|<\gamma$, for which

$$
\begin{aligned}
& \dot{w}(t)=P(t) w(t)+Q(t) v(t), \\
& {[C(\bar{p}(t), \bar{u}(t))+R(t) w(t)+S(t) v(t)]_{i} \leq-\alpha, \quad i=1,2, \ldots, l .}
\end{aligned}
$$

Let

$$
m \chi\left(\mathcal{T}_{\delta}\right)<1, \text { where } m:=\max \left\{\frac{\Delta}{1-\gamma}, \frac{1+\rho \Delta}{\alpha}\right\},
$$

where $\Delta$ and $\rho$ are as in (48). Then the feasibility mapping $f+F$ defined in (46) satisfies

$$
\operatorname{reg}(f+F ;(\bar{p}, \bar{u}) \mid 0) \leq\left(m^{-1}-\chi\left(\mathcal{T}_{\delta}\right)\right)^{-1} .
$$

Proof. Following the analysis in the beginning of this section, for any $A \in \mathcal{T}_{\delta}$ define the mapping

$$
\begin{aligned}
& (p, u) \mapsto G_{A}(p, u)(t) \\
& \quad=\left(\begin{array}{l}
-\dot{p}(t)+g(\bar{p}(t), \bar{u}(t))+P(t)(p(t)-\bar{p}(t))+Q(t)(u(t)-\bar{u}(t)) \\
C(\bar{p}(t), \bar{u}(t))+R(t)(p(t)-\bar{p}(t))+S(t)(u(t)-\bar{u}(t))
\end{array}\right)+\left(\begin{array}{l}
0 \\
\mathbb{R}_{+}^{l}
\end{array}\right) .
\end{aligned}
$$

Note that the term $\dot{p}$ is shifted from the second to the first summand in the right-hand side; here this is just for clarity.

Clearly, $G_{A}$ has a closed and convex graph. We will show that

$$
G_{A}(\bar{x}+\stackrel{\circ}{\mathbb{B}}) \supset c \stackrel{\circ}{\mathbb{B}} \quad \text { with } \quad c:=1 / m .
$$

Then the version of the Robinson-Ursescu theorem given in [12, Proposition 5B.2] together with the remark before [12, Exercise 5B.7] imply that $G_{A}$ is metrically regular at $\bar{x}=(\bar{p}, \bar{u})$ for 0 with modulus $m$. We have to verify that for every $y=(\xi, \nu)$ with $\|y\|<c$, the system

$$
\begin{aligned}
& \dot{p}(t)=\dot{\bar{p}}(t)+P(t)(p(t)-\bar{p}(t))+Q(t)(u(t)-\bar{u}(t))-\xi(t), \\
& C(\bar{p}(t), \bar{u}(t))+R(t)(p(t)-\bar{p}(t))+S(t)(u(t)-\bar{u}(t))-\nu(t) \leq 0
\end{aligned}
$$

has a solution $(p, u) \in W_{1, \infty}^{n, 0} \times L_{\infty}^{d}$ with $\|(p, u)-(\bar{p}, \bar{u})\|<1$. 
Fix $y=(\xi, \nu)$ as above. Let $(w, v)$ satisfy (49), and let $p$ be the solution of the differential equation in (53) corresponding to the control $u=v+\bar{u}$ and $p(0)=0$. Note that $p=w+\bar{p}-L \xi$ (see the paragraph before the statement of the theorem). From (50),

$$
\|(p, u)-(\bar{p}, \bar{u})\|=\|(w-L \xi, v)\| \leq \gamma+\Delta\|\xi\|<1 .
$$

Furthermore, from (49) and (50), skipping the dependence on $t$, we obtain

$$
\begin{aligned}
C(\bar{p}, \bar{u})+R(p-\bar{p})+S(u-\bar{u})-\nu & =C(\bar{p}, \bar{u})+R(w-L \xi)+S v-\nu \\
& \leq-\bar{\alpha}-R L \xi-\nu \leq 0,
\end{aligned}
$$

where $\bar{\alpha}:=(\alpha, \ldots, \alpha) \in \mathbb{R}^{l}$. Thus (52) holds.

Clearly, $F$ has a closed and convex graph. It remains to apply Corollary 19 (or Corollary 18 in the case of continuous differentiability) to obtain metric regularity of $f+F$ at $(\bar{p}, \bar{u})$ for 0 and the desired estimation of its modulus.

\section{REFERENCES}

[1] J.-P. Aubin and H. Frankowska, Set-Valued Analysis, Birkhäuser, Boston, 1990.

[2] J. M. Borwein AND D. M. ZhUAng, Verifiable necessary and sufficient conditions for openness and regularity of set-valued and single-valued maps, J. Math. Anal. Appl., 134 (1988), pp. 441-459.

[3] J. M. Borwein And Q. J. Zhu, Techniques of Variational Analysis, CMS Books Math., Springer, Berlin, 2006.

[4] R. Cibulka and A. L. Dontchev, A nonsmooth Robinson's inverse function theorem in Banach spaces, Math. Program. Ser A, 156 (2016), pp. 257-270.

[5] R. Cibulka And M. FABian, On primal regularity estimates for set-valued mappings, J. Math. Anal. Appl., 438 (2016), pp. 444-464.

[6] R. Cibulka and M. Fabian, A note on Robinson-Ursescu and Lyusternik-Graves theorem, Math. Program. Ser. B, 139 (2013), pp. 89-101.

[7] F. H. Clarke, On the inverse function theorem, Pacific J. Math., 64 (1976), pp. 97-102.

[8] F. H. Clarke, Optimization and Nonsmooth Analysis, Wiley, New York, 1983.

[9] F. H. Clarke, Functional Analysis, Calculus of Variations and Optimal Control, Grad. Texts in Math. 264, Springer, Berlin, 2013.

[10] A. L. Dontchev and W. W. Hager, An inverse mapping theorem for set-valued maps, Proc. Amer. Math. Soc., 121 (1994), pp. 481-489.

[11] A. L. Dontchev, The Graves theorem revisited, J. Convex Anal., 3 (1996), pp. 45-53.

[12] A. L. Dontchev and R. T. Rockafellar, Implicit Functions and Solution Mappings, 2nd ed., Springer, Berlin, 2014.

[13] A. V. Dmitruk, A. A. Milyutin, And N. P. Osmolovskil, Lyusternik's theorem and the theory of extremum (in Russian), Uspekhi Mat. Nauk, 35 (1980), pp. 11-46.

[14] M. Fabian, P. Habala, P. Hájek, V. Montesinos, and V. Zizler, Banach Space Theory: The Basis for Linear and Non-Linear Analysis, Springer, New York, 2011.

[15] M. Fabian And D. Preiss, A generalization of the interior mapping theorem of Clarke and Pourciau, Comment. Math. Univ. Carolin., 28 (1987), pp. 311-324.

[16] I. L. Glicksberg, A further generalization of the Kakutani fixed point theorem, with application to Nash equilibrium, Proc. AMS, 3 (1952), pp. 170-174.

[17] L. M. Graves, Some mapping theorems, Duke Math. J., 17 (1950), pp. 111-114.

[18] H. Hildebrand and L. M. Graves, Implicit functions and their differentials in general analysis, Trans. AMS, 29 (1927), pp. 127-153.

[19] A. D. Ioffe, Nonsmooth analysis: Differential calculus of nondifferentiable mappings, Trans. AMS, 266 (1981), pp. 1-56.

[20] A. D. Ioffe, Metric Regularity, Theory and Applications - A Survey, arXiv:1505.07920 [math.OC], 2015.

[21] A. F. Izmailov, Strongly regular nonsmooth generalized equations, Math. Program. Ser. A, 147 (2014), pp. 581-590.

[22] L. A. Lyusternik, On the conditional extrema of functionals (in Russian), Mat. Sbornik, 41 (1934), pp. 390-401.

[23] Z. PÁLES, Inverse and implicit function theorems for nonsmooth maps in Banach spaces, J. Math. Anal. Appl., 209 (1997), pp. 202-220. 
[24] J.-P. Рenot, Metric regularity, openness and Lipschitzian behavior of multifunctions, Nonlinear Anal., 13 (1989), pp. 629-643.

[25] J.-P. Penot, Calculus Without Derivatives, Grad. Texts in Math. 266, Springer, New York, 2013.

[26] B. H. Pourciau, Analysis and optimization of Lipschitz continuous mappings, J. Opt. in Theory Appl., 22 (1977), pp. 311-351.

[27] M. Renardy and R. C. Rogers, An Introduction to Partial Differential Equations, Springer, Berlin, 2004.

[28] S. M. Robinson, Strongly regular generalized equations, Math. Oper. Res., 5 (1980), pp. 43-62. 